\title{
Multi-Scale Turbulence Injector: a new tool to generate intense homogeneous and isotropic turbulence for premixed combustion
}

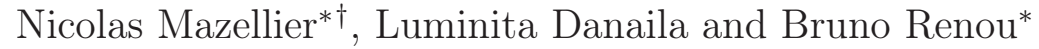 \\ UMR 6614 CORIA, INSA et Université de Rouen, BP 08, 76801 St. Etienne du Rouvray, France
}

\begin{abstract}
Nearly homogeneous and isotropic, highly turbulent flow, generated by an original multiscale injector is experimentally studied. This multi-scale injector is made of three perforated plates shifted in space such that the diameter of their holes and their blockage ratio increase with the downstream distance. The Multi-Scale Turbulence Injector (hereafter, MUSTI) is compared with a MonoScale Turbulence Injector (MoSTI), the latter being constituted by only the last plate of MuSTI. This comparison is done for both cold and reactive flows.

For the cold flow, it is shown that, in comparison with the classical mono-scale injector, for the MuSTI injector: (i) the turbulent kinetic energy is roughly twice larger, and the kinetic energy supply is distributed over the whole range of scales. This is emphasized by second and third order structure functions. (ii) the transverse fluxes of momentum and energy are enhanced, (iii) the homogeneity and isotropy are reached earlier $(\approx 50 \%)$, (iv) the jet merging distance is the relevant scaling length-scale of the turbulent flow, $(v)$ high turbulence intensity $(\approx 15 \%)$ is achieved in the homogeneous and isotropic region, although the Reynolds number based on the Taylor microscale remains moderate $\left(\operatorname{Re} e_{\lambda} \approx 80\right)$.

In a second part, the interaction between the multi-scale generated turbulence and the premixed flame front is investigated by laser tomography. A lean V-shaped methane/air flame is stabilised on a heated rod in the homogeneous and isotropic region of the turbulent flow. The main observation is that the flame wrinkling is hugely amplified with the multi-scale generated injector, as testified by the increase of the flame brush thickness.
\end{abstract}

Keywords: homogeneous and isotropic turbulence, multi-scale injection, premixed combustion.

\section{Introduction}

In the framework of turbulent premixed combustion, the morphology and the properties of the flame are essential inputs for combustion models. In particular, finite rate chemistry effects as well as flame front wrinkling and straining resulting from the competition between turbulence and combustion have to be accurately modeled to produce reliable predictions [1]. These requirements led to the concept of the so-called combustion diagrams (see e.g. [2] and [3]) which are derived from a phenomenological approach comparing the different time-scales and length-scales involved in the turbulence/combustion competition. Despite of their doubtless interest in combustion modeling, combustion diagrams are based on severe assumptions:

(i) the turbulence is considered as being non affected by heat release (frozen turbulence). However, the complex interaction between turbulent structures and the flame front (pre-heated and reaction zones) is not well interpreted by standard combustion diagrams [4].

(ii) the turbulent flow is considered as homogeneous and isotropic. This assumption allows to describe the turbulent flow properties using a reduced set of parameters (usually large-scale variables).

\footnotetext{
*Corresponding authors: nicolas.mazellier@univ-orleans.fr and bruno.renou@coria.fr

${ }^{\dagger}$ Permanent address: Institut PRISME, 8 rue Léonard de Vinci, 45072 Orléans, France
} 
(iii) criteria and regime limits are not determined from accurate estimations but rely on order-ofmagnitude approximations.

One of the objectives of works dedicated to turbulent premixed combustion was to improve the concept of combustion diagrams and therefore to lead to relevant combustion models. Experimentally, huge efforts were made to reproduce the different flame regimes. Unfortunately, this task requires to generate highly turbulent flows for which the homogeneity and isotropy assumptions are not fulfilled [5, 6, 7]. Numerically, recent advances in Direct Numerical Simulation (e.g. [8, 9]) provide a much deeper comprehension of the combustion physics by investigating the local flame structure. The confrontation of such approaches with reliable experimental data represents therefore a promising way to bring new insights in combustion modeling. From the experimental point of view, the main challenge is to conciliate highly turbulent level with homogeneity and isotropy properties.

Grid-generated turbulence, commonly recognised as nearly homogeneous and isotropic, has received a large attention over the past century since the precursory work of Batchelor \& Townsend [10]. Although this kind of flow has been extensively studied, it remains an ideal (and easy to be produced) candidate to identify and model the underlying physics of turbulence and combustion. Corrsin [11] provided a comprehensive review of grid-generated turbulence, which is produced by the passage of a stream through a grid with a specific pattern. The flow contraction imposed by the section reduction (with a blockage ratio $\sigma$ quantifying the ratio between the blockage area and the wind tunnel's section) at the grid location induces mean velocity gradients, implying turbulent kinetic energy production. Far away from the grid, turbulence decays and becomes nearly homogeneous and isotropic. By adding a secondary contraction downstream the grid, Comte-Bellot \& Corrsin [12] succeeded in improving global isotropy. The main disadvantage of standard grid-generated turbulence lies in its inability to reach high turbulence intensity (few percents) and therefore is restricted to moderate Taylor-based Reynolds numbers $R e_{\lambda}$.

Numerous attempts have been dedicated to produce homogeneous and isotropic turbulence at high Reynolds numbers. Gad-el-Hak \& Corrsin [13] developed a grid equipped with jets injecting air either in co-flowing or in counter-flowing configurations. They found that the counter-flowing configuration was able to produce a more intense turbulence (with $R e_{\lambda} \approx 150$ ) than the standard grid, by also keeping a good homogeneity and isotropy. In the nineties, Makita [14] and later Mydlarsky \& Warhaft 15] succeeded in generating highly turbulent flow using active grids $\left(R e_{\lambda} \approx 150-1000\right)$. Active grids are made of small wings mounted on a standard grid frame. The rotation of the wings is controlled by independent step motors which can be driven either in synchronous or random mode. Active grids produce an isotropy level which is slightly worse than that of the standard grid-generated turbulence. However, broad inertial ranges were revealed on energy spectra, attesting of the efficiency of such a device. More recently, Hurst \& Vassilicos [16] investigated turbulence generated by passive fractal grids made from the reproduction of a given pattern at different scales. For a square pattern, they reported high values of turbulence intensity $(8 \%)$, as well as large Reynolds numbers $\left(R e_{\lambda} \approx 150-450\right)$ although the blockage ratio of the fractal grids $(\approx 25 \%)$ is much lower than standard regular grids $(\approx 34 \%)$. The isotropy level was comparable to that of active grids and the homogeneous and the isotropic region appeared further away than for the standard grid-generated turbulence.

The purpose of the present work is to create experimentally a nearly homogeneous isotropic turbulence, with a large turbulence intensity. We investigate an original turbulence generator made of the combination of several perforated plates. It is worth to mention that turbulence manipulation by grid/screen combination is not a new concept. Tan-Atichat \& al. [17] and later on Groth \& Johansson [18] investigated the turbulence reduction with combination of screens and perforated plates. The efficiency of their turbulence "manipulators" was tested for various upstream conditions. They found that 
the energy decay was accelerated through the interaction between the incoming turbulence and the small-scale turbulence generated by the "manipulators". However, to our knowledge, no attempt has been devoted to amplify turbulence by using grid/screen combination, which is the aim of the present work.

The paper is organized as follows. The experimental facility and the measurement techniques are described in section 2. The characteristics of both the original multi-scale injector and a reference injector are presented in section 3. The main properties of turbulence at large- and small-scales, as well as the interaction with premixed combustion are discussed in section 4 .

\section{The experimental set-up}

\subsection{The wind-tunnel facility}

Experiments were carried out in an open-loop vertical wind-tunnel with a square test section $\left(8 \times 8 \mathrm{~cm}^{2}\right)$ adapted to the study of steady combustion [19], see Figure 1. The test section is $40 \mathrm{~cm}$ long and is equipped with optical access. In non-reactive configuration, the working fluid is air supplied by a network of compressed air, regulated via a mass flow meter/controller Bronkhorst (F-206AI).

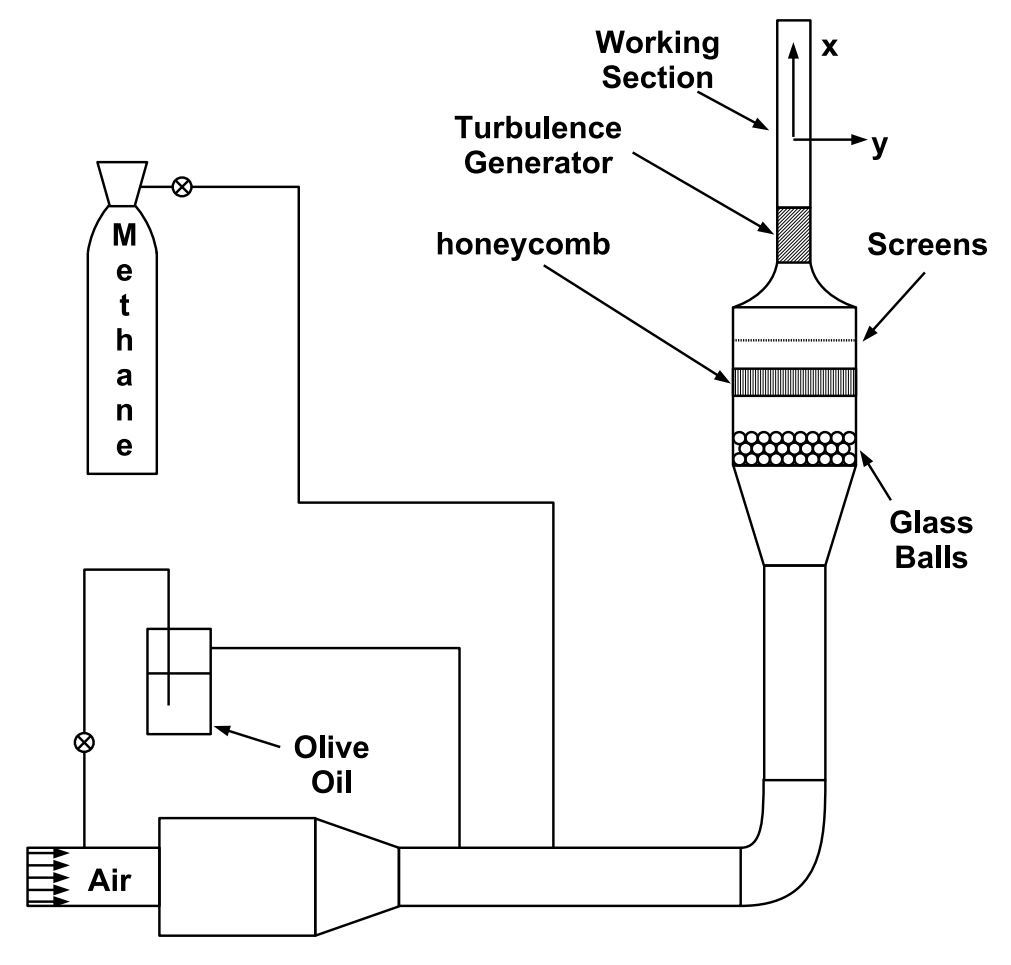

Figure 1: Schematic representation of the wind-tunnel facility.

The flow is directed to a divergent-convergent settling chamber constituted of glass balls bed, honeycombs and screens to attenuate residual turbulent perturbations. The flow is then accelerated through a (11:1) aspect ratio contraction. The turbulence generators are set between the outlet of the convergent and the inlet of the working section. In the absence of any obstacle, the residual turbulence level is as low as $0.4 \%$ in the working section. The inlet velocity $U_{\infty}$ computed from the mean flow rate and the test section area is imposed to $3.7 \mathrm{~m} / \mathrm{s}$. 
In the following, we use the notation $(u, v, w)$ for denoting the velocity components in the $(x, y, z)$ directions. The origin of the streamwise direction $x$ is taken at the outlet of the turbulence generator.

\subsection{Velocity measurements}

Velocity field is investigated by two independent optical methods: Particle Image Velocimetry $(P I V)$ and Laser Doppler Velocimetry $(L D V)$. A schematic representation of the experimental set-up is given in Figures 2(a) and 2(b), During measurements, the wind-tunnel is seeded with olive oil droplets generated by a particle-seeding apparatus and injected far upstream of the working section. The average diameter $d_{p}$ of the olive oil particles is close to $1 \mu \mathrm{m}$, as separately calibrated with a Malvern diffractometer. The Stokes number $S_{k}=\tau_{p} f_{\eta}$ comparing the particle response time $\tau_{p}=\frac{d_{p}^{2}}{18 \nu}$ (with $\nu$ the kinematic viscosity of the fluid) and the Kolmogorov's frequency $f_{\eta}=\frac{U}{2 \pi \eta}$ (with $\eta$ the Kolmogorov scale and $U$ the mean flow velocity), is much lower than 0.1 ensuring a good flow tracing. The seeding rate was adjusted to ensure good droplet density and homogeneity during optical measurements.
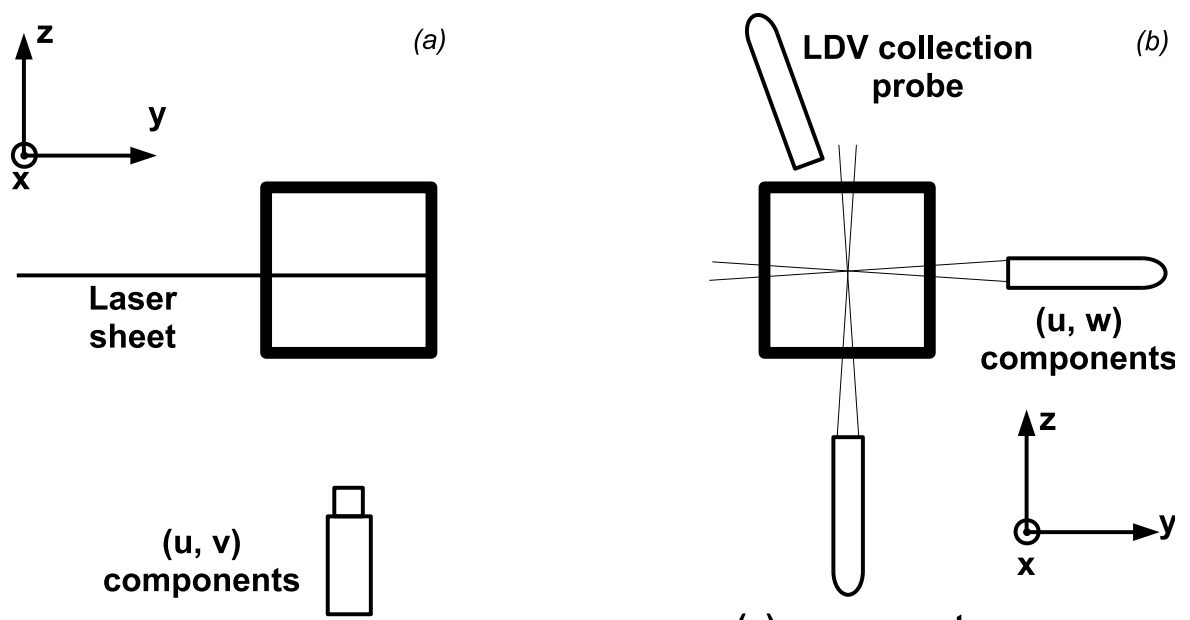

(v) component

Figure 2: Top-views of the PIV (a) and the $L D V$ (b) configurations.

\subsubsection{PIV system}

The spatial velocity field in non-reactive configuration is investigated via PIV with a Nd-Yag laser (Big Sky laser, $120 \mathrm{~mJ} /$ pulse, $532 \mathrm{~nm}$ ) as light source. The vertical laser sheet coincides with the middle plane of the working section corresponding to the $(x, y)$-plane (see Figure 2(a)). Light scattered from olive oil droplets is collected on a CCD camera (FlowMaster LaVision, 12-bits, $1280 \times 1024$ pix $^{2}$ ) with a $50 \mathrm{~mm} \mathrm{f} / 1.2$ Nikkor lens. The magnification ratio is of $16 \mathrm{pix} / \mathrm{mm}$, which leads to a physical field of view of $80 \mathrm{~mm} \times 64 \mathrm{~mm}$. Particle images are post-processed with the standard commercial package available in Davis 6.2 (LaVision Company). Velocity field computation is based on a multi-pass algorithm with adaptive window deformation. The starting and final interrogation windows are imposed to 64pix ${ }^{2}$ and $16 \mathrm{pix}^{2}$ respectively with $50 \%$ overlapping giving a final map of $160 \times 128$ vectors. According to [20], the spatial resolution of the PIV system is about $2.25 \mathrm{~mm}$. We estimate that the final interrogation window ranges between 10 and 25 times bigger than the Kolmogorov scale $\eta$. Due to the poor spatial resolution, PIV results will be used to investigate the large scales of the flow. To ensure statistical convergence, a total of 3500 images are acquired for each configuration. Both optics and camera are mounted on 
a 3D traversing system allowing to displace the investigated frame along the entire working section. An overlap of almost 1/3 between the different frames is imposed in order to check the continuity of statistics.

\subsubsection{LDV system}

Three-component $L D V$, with a $8 W$ Argon-ion laser $(514.5 \mathrm{~nm}, 488 \mathrm{~nm}$ and $476.5 \mathrm{~nm})$ as light source, is used to measure local velocity statistics with a good temporal resolution. Data are collected in forward scatter mode at $25^{\circ}$ off axis (see Figure 2(b) and processed with an IFA755 processor (TSI) set in a non-coincident single measurement per burst mode. Signals are then digitized and stored on computer hard-drive via ICA-LDV32.net software. For each measurement, several parameters (laser intensity, photomultiplier intensity, filtering window ...) are carefully adjusted to maximize the mean data rate $f_{s}$ which ranges between $12 \mathrm{kHz}$ and $22 \mathrm{kHz}$. The minimum sampling rate $f_{s}$ varies between 0.6 and 2.6 times the estimated Kolmogorov's frequency $f_{\eta}=\frac{U}{2 \pi \eta}$. Each optic fiber is clamped on independent high precision linear stages allowing to superimpose accurately the three measurement volumes. The $L D V$ spatial resolution $\frac{\ell}{\eta}$ (with $\ell$ the typical size of the $L D V$ measurement volume) is estimated to range between 0.6 and 1.8, which is at least 10 times better than $P I V$. For each station, $8 \times 10^{6}$ samples are throughout acquired (almost $1 / 3$ per channel). The associated measurement time ranges between $5 \times 10^{4}$ and $25 \times 10^{4}$ integral time-scale ensuring the statistical convergence. The $L D V$ system is mounted on a 3D traversing system controlled by computer. Measurement points are taken along the tunnel centreline and downstream the centre between two successive holes.

\subsection{Flame structure characterization}
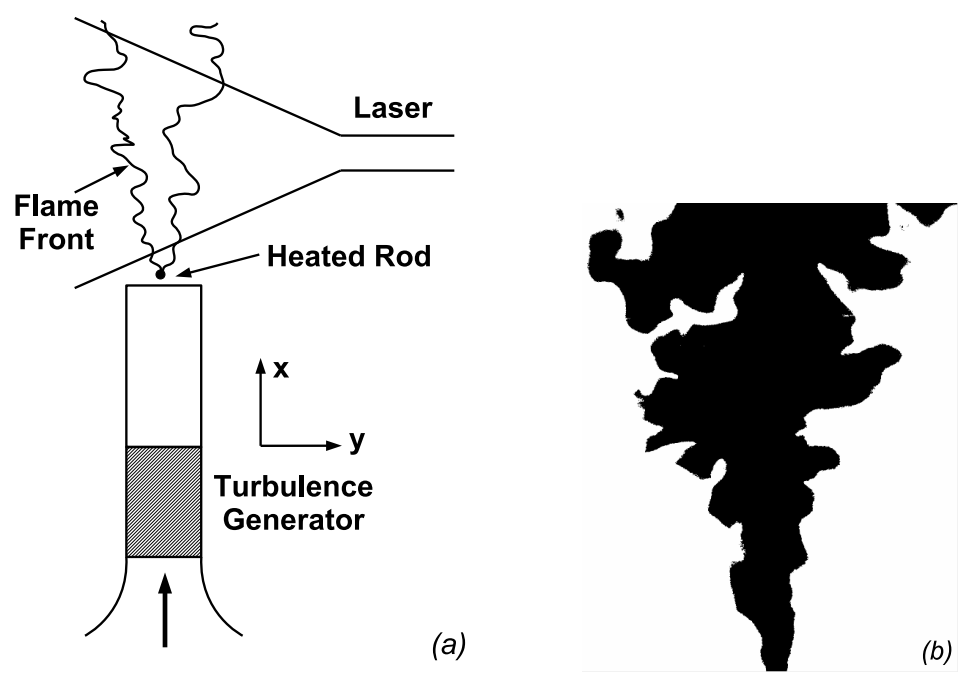

Figure 3: (a) Front-view of the Tomography configuration. (b) Typical example of binary flame front image obtained by thresholding.

In reactive configuration, methane/air mixture is used with an equivalent ratio $\phi=0.6$. A 2D stationary $\mathrm{V}$-shaped flame is stabilised on a tiny heated rod ( $1 \mathrm{~mm}$ in diameter) placed in the middle plane of the test section (see Figure 3(a) . The heated rod position, denoted $x_{\text {rod }}$, is located in the homogeneous and isotropic region of the turbulent flow. Downstream the rod location, the lateral walls of the tunnel are removed to avoid flame/wall interaction. In order to minimize the effect of lateral 
mixing layers on flame properties, we focus on the very near-field of the heated rod. The flames are visualised by laser sheet tomography with the same light source as for PIV. The reacting flow was seeded with olive oil droplets which evaporate at the entrance of the flame front. The instantaneous two-dimensional flame surface was obtained by differentiation, on the flame recordings, of the dark and bright areas, representing burned and unburned states respectively. The flame images are recorded with a PIV camera using identical magnification ratio with that of the PIV. For each image, the contours of the turbulent flame (which physically correspond to the instantaneous location of the isotherm 500K) are extracted with an edge detection algorithm using an adaptive and smoothing procedure. Figure 3(b) shows a typical example of the resulting binarized image where white areas correspond to fresh gases, while black regions represent the burnt gases (and therefore the flame). A total of 1000 images were acquired to determine the Reynolds average properties of the flame.

\section{The turbulence generators}

\subsection{The reference injector}

In the present work, turbulence is generated by perforated plates either alone or in combination via a multi-scale injector which is the novelty of the paper. Each plate is characterised by both a hole diameter $D$ and the mesh size $M$ and spans the entire wind tunnel. The holes are circular and arranged in a triangular network as shown in Figure 4(a). Moreover, the perforation is straight over the entire thickness of the plates and the holes network is chosen such that the tunnel's centreline coincide with a hole centre. A typical illustration of a perforated plate is given in Figure 4(b).
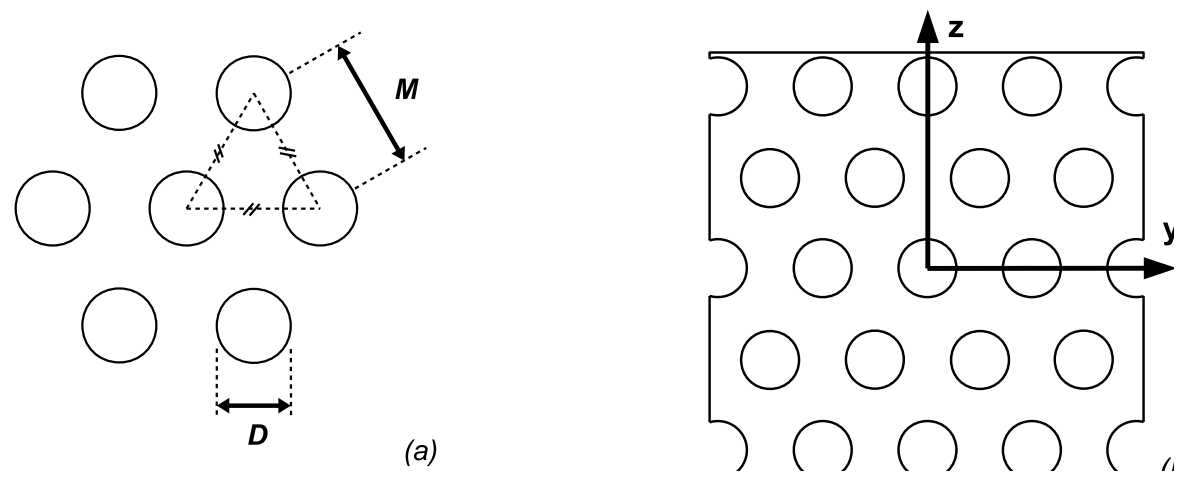

Figure 4: (a) Hole pattern. (b) Typical example of perforated plate.

The amount of turbulent kinetic energy injected by each plate is controlled by the pressure drop $\Delta p[23]$ :

$$
\Delta p=\frac{1}{2} f(R e) K_{\sigma} \rho U^{2}
$$

with $\rho$ the fluid density, $U$ the mean velocity in the $x$ direction, $f(R e)$ an empirical function and

$$
K_{\sigma}=\frac{1-(1-\sigma)^{2}}{(1-\sigma)^{2}}
$$

The function $f(R e)$ becomes constant ( $\approx 0.5$ for screens) for high enough Reynolds number [18].

Prior to the manufacturing of the new multi-scale device, a single perforated plate was chosen and used as a reference injector. The geometrical properties of this reference injector are given in Table 1 . 
By opposition to the multi-scale injection, the reference injector produces a mono-scale forcing of the flow and relies on the so-called turbulent energy cascade to transfer the injected energy from large-scales to small-scales. The reference injector is therefore called MoSTI (Mono-Scale Turbulence Injector) in the following.

\begin{tabular}{cccc}
\hline$D(\mathrm{~mm})$ & $M(\mathrm{~mm})$ & $\sigma$ & $K_{\sigma}$ \\
\hline \hline 15 & 24.7 & 0.67 & 8.18 \\
\hline
\end{tabular}

Table 1: Geometrical characteristics of the mono-scale injector (MoSTI)

Considering technical constraints (tunnel's size for instance), the mesh size $M$ has been fixed to about $1 / 3$ of the tunnel side. The blockage ratio, $\sigma=0.67$, is situated at midway between that usually reported for bi-plane grids $(\sigma \approx 0.34)[12]$ and that used by Villermaux \& Hopfinger $(\sigma \approx 0.9)[22]$. The latter observed sustained jet-oscillations over large distance downstream the grids that are not present in our study.

\subsection{The multi-scale injection concept}

The multi-scale injector we have designed is built from a combination of $N$ perforated plates. In the present work, for simplicity we have chosen only three plates $(N=3)$. Moreover, one of them is the same as that for the MoSTI.

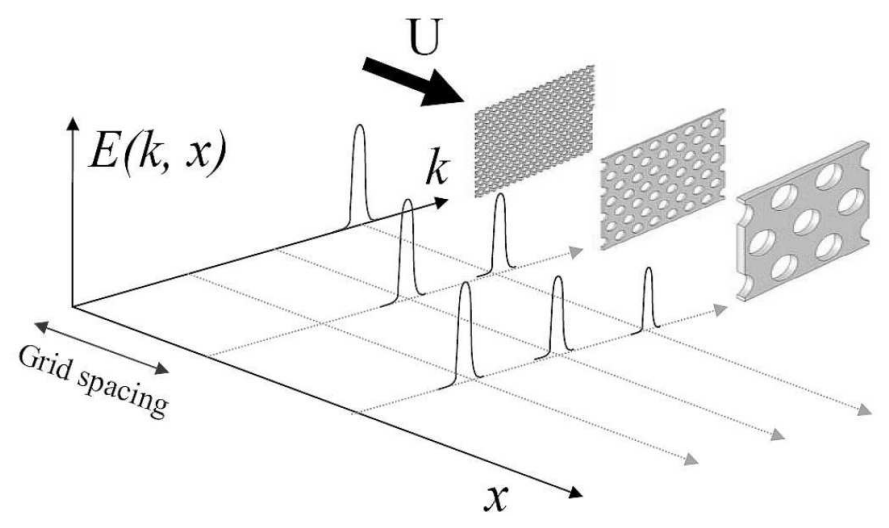

Figure 5: Schematic representation of the Multi-Scale injector (MuSTI).

As shown in Figure 5, the three plates are shifted in space such that both hole's diameter $D_{j}$ and mesh size $M_{j}$ increase in the mean-flow direction (where subscript refers to the $j^{\text {th }}$ plate). This device generates a space-delayed (or time-delayed) multi-scale forcing of the flow and is therefore refered to as MuSTI (Multi-Scale Turbulence Injector) in the following. The concept of this multi-scale injection can be easily represented in wavenumber space (see Figure 5). Following the flow, small-scales (high wavenumbers) are first excited, then medium-scales and finally large-scales (low wavenumbers). Using this arrangement, we aim to experimentally produce a multi-scale forcing in wavenumber space, thus mimicking a cascade process. In other words, each perforated plate $j$ is expected to inject a specific spectral energy $E_{j}(k)$ at given injection wavenumbers $k$. This approach has already been developed, for instance, by Mazzi \& Vassilicos [21] who performed continuous and discrete fractal forcing in stationary Direct Numerical Simulations. The authors revealed that the turbulence generated was very sensitive to 
the properties of the forcing. From Figure 5, one can remark that the complete design of the multi-scale injector requires three independent geometrical parameters:

1. the mesh size $M_{j}$,

2. the spacing $L_{j}$ between plates $j$ and $j+1$,

3. the pressure drop constant $K_{\sigma j}$.

It is important to notice that the hole's diameter $D_{j}$ and the blockage ratio $\sigma_{j}$ are functions of both $M_{j}$ and $K_{\sigma j}$.

\subsection{The final design of the multi-scale injector}

The geometrical parameters of the last perforated plate being fixed (identical to those of the MoSTI), it remains to choose those of the two first plates. The approach we have adopted to choose the triad $\left(M_{j}, K_{\sigma j}\right.$ and $\left.L_{j}\right)$ of those plates has been based on the investigation of the turbulence generated downstream by various perforated plates used alone.

\subsubsection{The mesh size $M_{j}$}

The mesh size $M_{j}$ controls the typical length-scale of energy-contained structures generated downstream the plate $j$. This is emphasized by Figure 6(a) showing the streamwise variation of the longitudinal integral length-scale $\Lambda_{u}\left(\equiv \int_{0}^{\infty} R_{u u}(h) d h\right.$ with $R_{u u}$ the longitudinal velocity autocorrelation coefficient and $h$ the spatial lag recovered from Taylor's hypothesis) obtained from $L D V$ measurements, downstream from various individual plates. It is therefore reasonable to assimilate $M_{j}$ to the preferential injection wavenumber $k$, i.e. $k \sim M_{j}^{-1}$.

In the present work, the smallest mesh size $M_{1}$ of the MuSTI corresponds to the Taylor micro-scale of the turbulence generated by the MoSTI, whilst the intermediate mesh size $M_{2}$ corresponds to a characteristic scale of its inertial range.
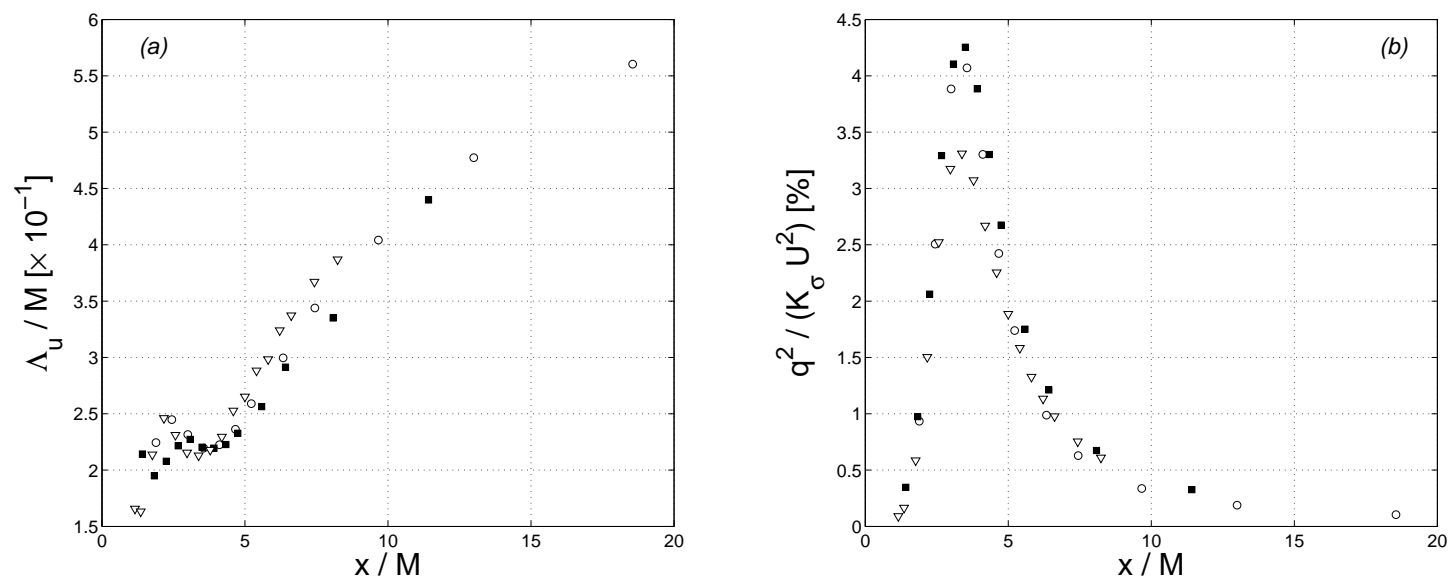

Figure 6: Streamwise evolution of (a) the dimensionless integral length-scale $\Lambda_{u} / M$ and (b) the dimensionless turbulent kinetic energy $q^{2} /\left(K_{\sigma} U^{2}\right)$ measured on the wind-tunnel's centreline downstream various perforated plates used separately. Symbols: $\bigcirc, D=6 \mathrm{~mm}$ and $M=9 \mathrm{~mm}(R e=1480)$; $D=8.5 \mathrm{~mm}$ and $M=12 \mathrm{~mm}(R e=2100) ; \nabla, D=15 \mathrm{~mm}$ and $M=24.7 \mathrm{~mm}(R e=3700)$. 


\subsubsection{The plate spacing $L_{j}$}

The plate spacing $L_{j}$, which fixes the turbulence state, has to be chosen carefully. Indeed, $L_{j}$ has to be neither too short, in order to give time to turbulent structures generated by plate $j$ to have strong enough turbulence passing through the plate $j+1$, nor too long, to avoid the complete dissipation of those structures. The evolution of the dimensionless turbulent kinetic energy $q^{2}\left(\equiv\left\langle u^{2}\right\rangle+\left\langle v^{2}\right\rangle+\left\langle w^{2}\right\rangle\right)$ measured with $L D V$ indicates that the best compromise is to set the plate $M_{j+1}$ at the energy peak location of the plate $M_{j}$, i.e. $L_{j} \approx 3.5 M_{j}$ for this particular kind of plates (see Figure 6(b)). This distance has been therefore used as the separation between two successive plates for the MuSTI.

\subsubsection{The pressure loss coefficient $K_{\sigma j}$}

Confronting the results of both Figures 6(a) and 6(b) suggests that the individual energy spectrum $E_{j}(k)$ (defined by $q^{2}=\int_{0}^{\infty} E_{j}(k) d k$ ), induced by each individual plate, can be written under the following form, without loss of generality:

$$
E_{j}(k) \sim K_{\sigma j} M_{j} g_{j}\left(k M_{j}\right)
$$

where $g_{j}\left(k M_{j}\right)$ is a dimensionless function representing the shape of the energy spectrum in the wavenumber space. As mentioned previously, the goal of the MuSTI device is to reproduce a cascade process which can be expressed through a power-law:

$$
E(k)=\sum_{j} E_{j}(k) \sim k^{-\gamma}
$$

with $\gamma$ the power-law exponent. Combining Equations (3) and (4) leads therefore to:

$$
K_{\sigma j} \sim M_{j}^{\gamma-1}
$$

This relation implies therefore that the plates constituting the multi-scale injector are self-similar. The diameter $D_{j}$ is then simply obtained from the values of both $M_{j}$ and $K_{\sigma j}$ for each plate. In the present work, the power-law exponent $\gamma$ that we have used is equal to 1.57 which is very close to the well-known 5/3 exponent reported for 3D turbulent flows [24].

\subsubsection{The MuSTI device}

Following the steps mentioned hereinbefore, the complete design of the MuSTI device has been achieved. Its geometrical properties are reported in Table 2

\begin{tabular}{cc|cc|cc|cc}
\hline$D_{1}(m m)$ & 3 & $M_{1}(m m)$ & 4 & $\sigma_{1}$ & 0.49 & $K_{\sigma 1}$ & 2.84 \\
$D_{2}(m m)$ & 6 & $M_{2}(m m)$ & 9 & $\sigma_{2}$ & 0.60 & $K_{\sigma 2}$ & 5.25 \\
$D_{3}(m m)$ & 15 & $M_{3}(m m)$ & 24.7 & $\sigma_{3}$ & 0.67 & $K_{\sigma 3}$ & 8.18 \\
\hline
\end{tabular}

Table 2: Geometrical characteristics of the multi-scale injector (MuSTI).

The direct comparison between the mono-scale and the multi-scale injectors leads to a couple of remarks that it is important for the reader to notice and keep in mind:

1. due to pressure loss addition, the total amount of energy injected by the $M u S T I$ is roughly twice that of the MoSTI $\left(K_{\sigma}(M u S T I)=\sum_{j} K_{\sigma j}=16.27\right)$. Inverting the relation given in Equation 
(2) implies that the equivalent blockage ratio of the $M u S T I$ injector $\sigma(M u S T I)$ is about $13 \%$ higher than that of the MoSTI device.

2. for the $M U S T I$ injector, the genesis of the turbulence starts about $45 \mathrm{~mm}\left(=3.5 M_{1}+3.5 M_{2}\right)$ upstream from the wind tunnel's inlet. As we show in the following, this property will strongly impact the development of the turbulence downstream the injector.

Moreover, we stress that care should be taken in extrapolating the results we present in the following section as the design of the MuSTI has required to fix several degrees of freedom ( $\gamma$ for instance). Indeed, one can expect that those variables are of fundamental importance in the development of turbulence downstream the multi-scale injector.

In the following, for sake of simplicity, the largest mesh size $M_{3}=24.7 \mathrm{~mm}$ is used as a reference length-scale for both injectors.

\section{Results and discussion}

\subsection{The mean flow}

In the near-field of individual perforated plates, jets issuing from holes, extend via lateral spreading and merge at a distance $L_{m}$ from the plate [22]. A schematic view of the flow developing in the neighborhood of an individual perforated plate is given in Figure 7

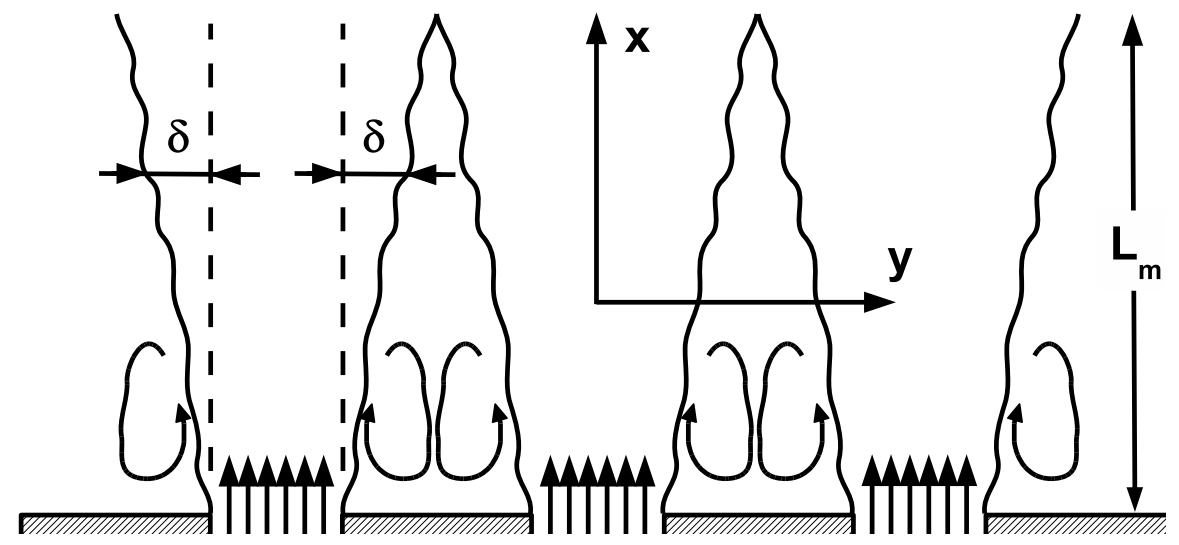

Figure 7: Schematic representation of the near-field of an individual perforated plate.

This sketch compares very well with the map of scaled mean velocity $U(x, y) / U_{\infty}$ in the lee of the $M o S T I$ injector given in Figure 8(a). One can remark the presence of recirculating bubbles before the jet merging as evidenced by the average streamlines.

On the contrary, the average streamlines computed for the MuSTI injector (Figure 8(b) do not evidence recirculating bubbles between issuing jets, at least downstream $x / M_{3}=1.15$ (we did not perform PIV measurements further upstream because of laser light reflexions). This result is the first illustration of the influence of the multi-scale injection onto the flow development. Furthermore, one can notice that the normalized mean velocity field $U(x, y) / U_{\infty}$ is quite different in intensity (i.e. colour) between the two experiments (the same colorbar is used for both injectors).

This observation is highlighted by the spanwise profiles of $U / U_{\infty}$ plotted in Figure 9 (a) for $x / M_{3}=$ 1.15. The profile obtained for the MoSTI injector is characteristic of a potential core (plateau around 

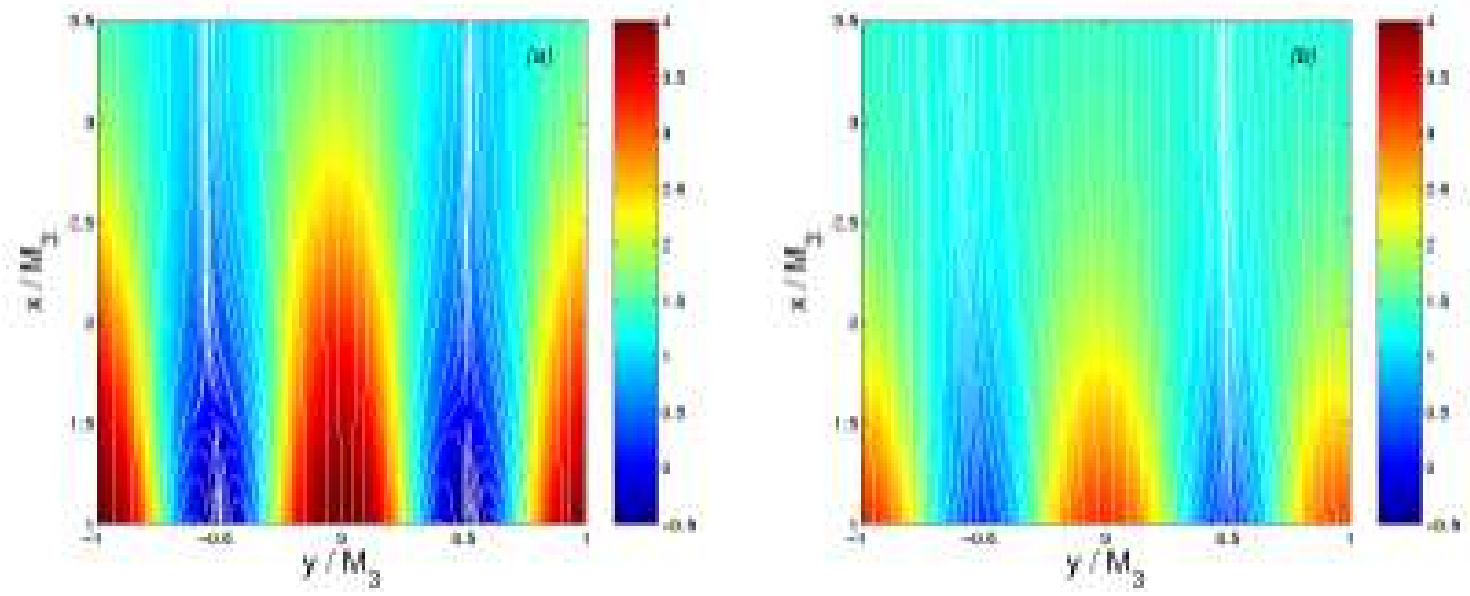

Figure 8: Average streamlines and normalized streamwise mean velocity map $U(x, y) / U_{\infty}$ (coloured background) in the lee of (a) the MoSTI injector and (b) the MuSTI injector computed from PIV measurements.

the centreline) surrounded by shear-layers. The latter are responsible for the lateral jet spreading as shown by the jet expansion compared to the hole's diameter. One can also remark that the presence the recirculating bubbles induces negative velocities on the jet boundaries.
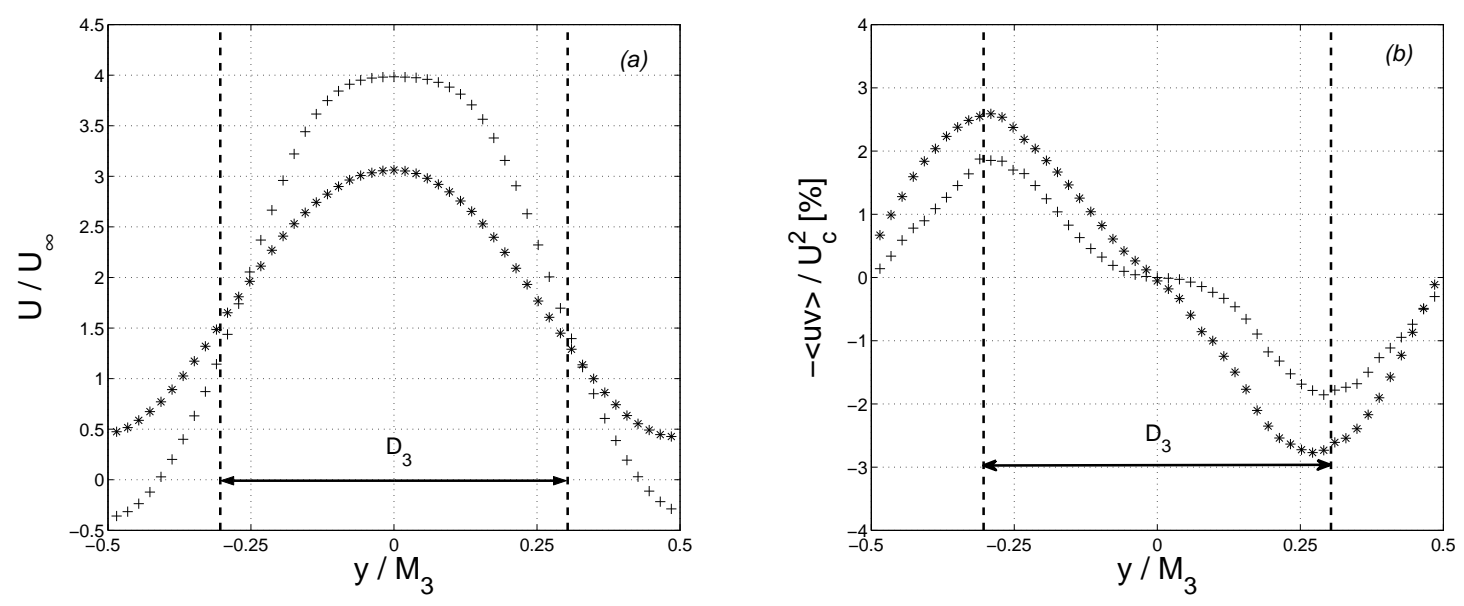

Figure 9: (a) Dimensionless mean velocity profiles (central hole) measured at $x / M_{3}=1.15$ with $P I V$. (b) Dimensionless transverse velocity flux measured from PIV at the same location for both injectors. Symbols: +, MoSTI injector; ${ }^{*}, M U S T I$ injector. The central hole position is illustrated by the vertical dashed lines.

The jet spreading downstream the MuSTI injector is clearly larger than that of the MoSTI injector. This behaviour reflects that the lateral shear-layer thickness $\delta$ induced by the multi-scale injection is larger (by about $25 \%$ at this location) than that of the reference device. Invoking mass-conservation principle, this lateral expansion enhancement is followed by a decrease of the centreline velocity $U_{c}=$ $U(x, y=0, z=0)$ for the MuSTI injector.

This fast expansion of the shear-layers is related to the increase of the transverse velocity flux $\langle u v\rangle$ as illustrated in Figure 9(b), The jet spreading can be interpreted through the simple model of turbulent 
viscosity $\nu_{t}$ which acts as an effective diffusion coefficient:

$$
\langle u v\rangle=-\nu_{t} \frac{\partial U}{\partial y}
$$

Due to the anticipated genesis of turbulence through small-scale injection, the MuSTI device accelerates the exchange of energy in all directions increasing therefore the transverse fluxes in comparison with the mono-scale injector. As a consequence, the turbulent viscosity produced by the MuSTI is about two times bigger than that of the MoSTI.

\subsection{Homogeneity and isotropy}

One of the main objectives of the present work is to generate a turbulent flow as nearly as possible homogeneous and isotropic. As shown hereinbefore, the MuSTI enhances the transverse velocity flux which are of major importance for the redistribution of energy and by the way for homogeneity and isotropy. It is obvious from Figure 7 that the homogeneity condition can only be satisfied beyond the jet merging position symbolised by the length $L_{m}$. This distance is straightly dependent on the shear-layers expansion rate and is reached when $\delta / M_{3} \sim 1$. The shear-layer spreading results from the competition between convection and turbulent diffusion characterised by the time-scales $\tau_{\text {conv }} \sim x / U$ and $\tau_{\text {diff }} \sim \delta^{2} / \nu_{t}$ respectively. Assuming that both time-scales are of the same order of magnitude, it then comes:

$$
\frac{\delta}{x} \sim R e_{t}^{-1 / 2}
$$

with $R e_{t}=\frac{U x}{\nu_{t}}$ a turbulent Reynolds number. The jet merging conditions $\left(\delta=M_{3} / 2\right.$ and $\left.x=L_{m}\right)$ leads therefore to:

$$
\frac{M_{3}}{L_{m}} \sim \sqrt{\frac{\nu_{t}}{U L_{m}}}
$$

This equation illustrates the influence of the turbulent viscosity in the jet merging phenomenon. The latter should therefore be accelerated when the turbulent viscosity is increased.

The jet merging distance $L_{m}$ has been experimentally estimated by comparing the streamwise evolution of the relative difference between the centreline mean velocity $U_{c}(x)$ and the minimum mean velocity $U_{\min }(x)$ (corresponding to the centre between 2 successive holes, i.e. $y=M_{3} / 2, z=0$ ). For this purpose, we introduce the dimensionless ratio $H$ such that:

$$
H=\frac{U_{c}-U_{\min }}{U_{c}}
$$

It is worth to notice that $H$ is a representative criterion to evaluate the flow homogeneity in the $y-z$ plane. Figure $10(\mathrm{a})$ shows the streamwise variation of the ratio $H$ for both injectors. On this plot, the comparison of the PIV and $L D V$ measurements shows a very good agreement.

As expected, in the lee of the injectors, the high values of $H$ reflect the flow inhomogeneity in the transverse direction. Further downstream, these inhomogeneities are smoothed due to the shear-layer expansion resulting in the decrease of $H$ which tends towards zero far away from the plate. One can remark that the transverse inhomogeneity is systematically smaller (tending therefore more rapidly towards zero) for the MuSTI injector. We define the merging distance $L_{m}$ as the position where the ratio $H$ becomes smaller than $10 \%$. This value is illustrated in Figure 10(a) by the horizontal dashed line. The dimensionless merging distance $L_{m} / M_{3}$ is found equal to $5.35 \pm 0.25$ and $3.50 \pm 0.02$ respectively for MoSTI and MuSTI injectors. It is important to notice that rescaling the streamwise 

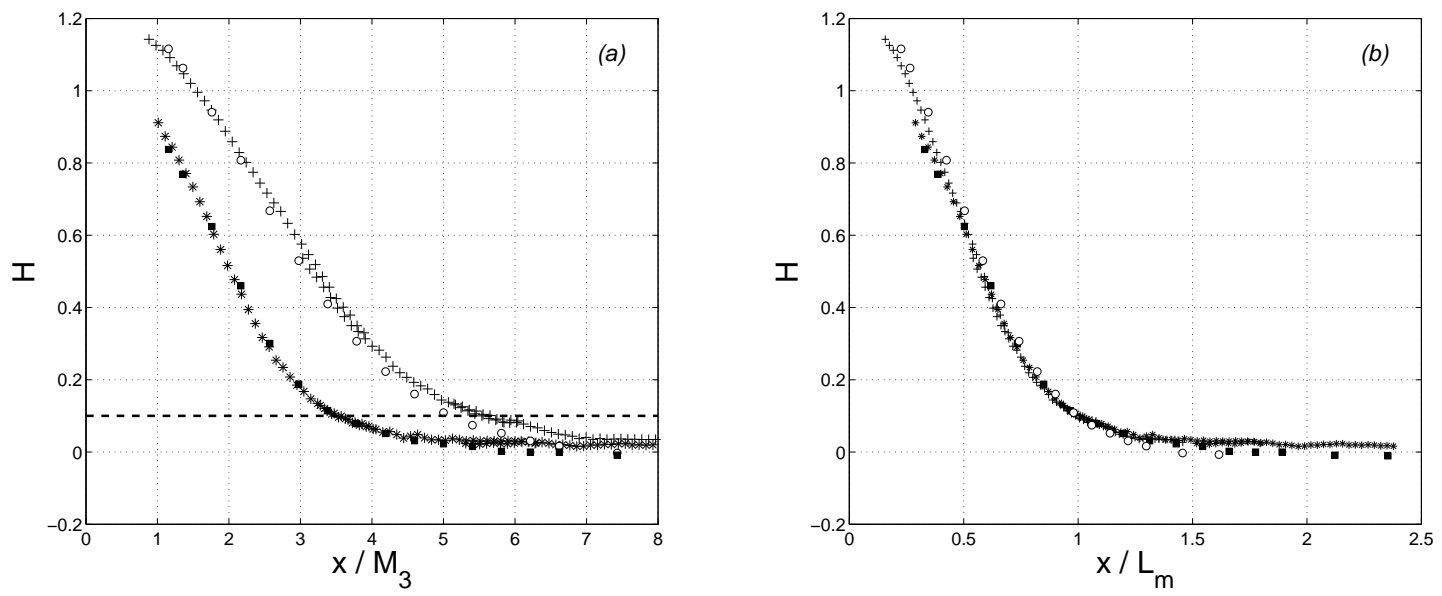

Figure 10: Evolution of the ratio $H$ as a function of the streamwise distance $x$ scaled with the mesh size $M_{3}(a)$ and with the merging distance $L_{m}(b)$. The dashed line represents the merging location $L_{m}$. Symbols: +, MoSTI injector (PIV measurements); *, MuSTI injector (PIV measurements); $\bigcirc$, $M o S T I$ injector ( $L D V$ measurements); $\mathbf{\square}, M u S T I$ injector ( $L D V$ measurements).

distance $x$ by the merging length $L_{m}$ permits to collapse very well the ratio $H$ for both injectors as shown in Figure 10(b).

The global isotropy is commonly evaluated via the factor $I$ defined by:

$$
I=\sqrt{\frac{\left\langle u^{2}\right\rangle}{\left\langle v^{2}\right\rangle}}
$$

Figures 11(a) and 11(b) show the streamwise variation of the global isotropy ratio $I$ computed on the tunnel's centreline for both injectors. As expected, the flow is strongly anisotropic close to the injector and then tends to isotropy further away. The MuSTI injector is characterised by a better global isotropy ratio $I$ than the MoSTI device. Beyond $x / L_{m}=1$, the global isotropy level obtained for the MuSTI injector is comparable to those reported by Comte-Bellot \& Corrsin [12] for different kind of regular grids without secondary contraction. Moreover, this level is better than the values reported by Mydlarski \& Warhaft [15] for active grids $(\approx 1.2)$ and Hurst \& Vassilicos [16] for fractal grids (between $\approx 1.2$ and $\approx 1.4$ depending on the grid pattern).

From these quantitative results three conclusions arise:

(i) the multi-scale injection strongly reduces the inhomogeneous and anisotropic region downstream the injector.

(ii) the nearly homogeneous and isotropic region appears much faster (less than $5 M_{3}$ ) than in standard grid-generated turbulence ( $40 M$ for low-blockage regular grid for instance)

(iii) the merging length $L_{m}$ is revealed as a typical scaling length for the two first order moments.

\subsection{The one-point kinetic energy budget}

The second objective of our new multi-scale injector is to generate intense turbulence level in the nearly homogeneous and isotropic region. This property can be evaluated via the dimensionless turbulent kinetic energy $q^{2} / U_{c}^{2}$ measured on the tunnel's centreline. 

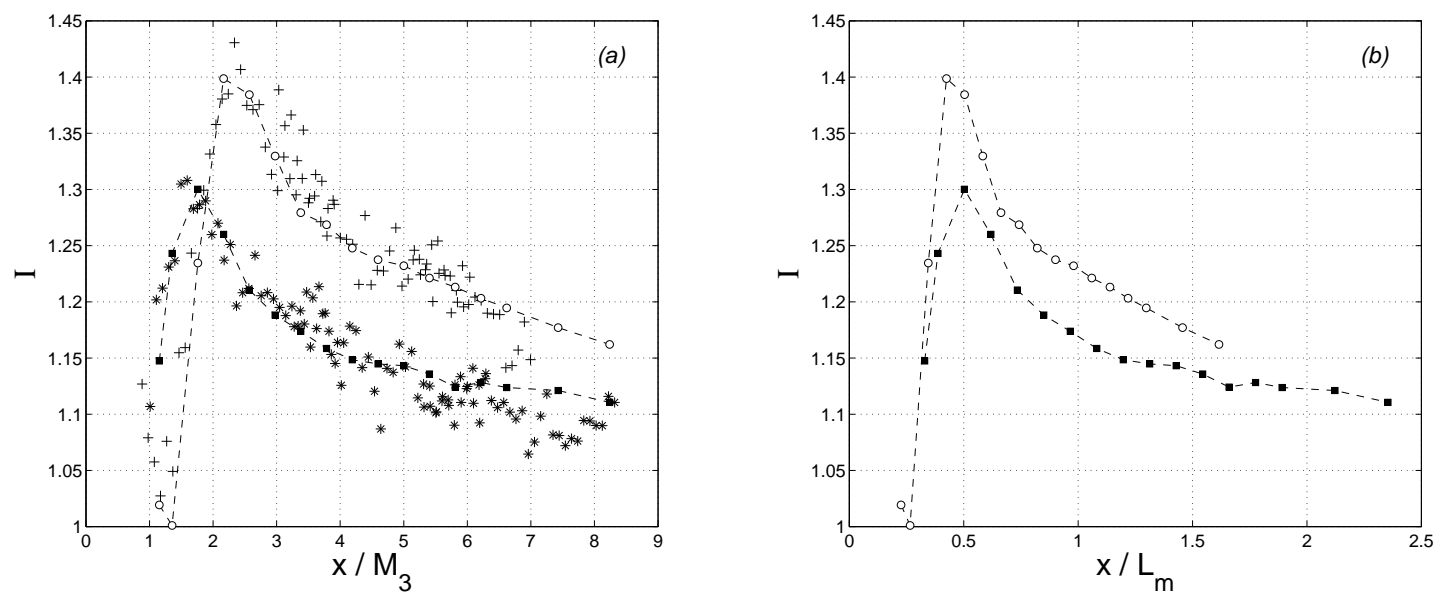

Figure 11: Streamwise evolution of the global isotropy factor $\sqrt{\left\langle u^{2}\right\rangle /\left\langle v^{2}\right\rangle}$ with respect to the streamwise distance $x$ scaled by the plate's mesh size $M_{3}(a)$ and by the merging length $L_{m}(b)$. Symbols: + , MoSTI injector (PIV measurements); *, MuSTI injector (PIV measurements); $\bigcirc$, MoSTI injector ( $L D V$ measurements); $\mathbf{\square}, M u S T I$ injector ( $L D V$ measurements).
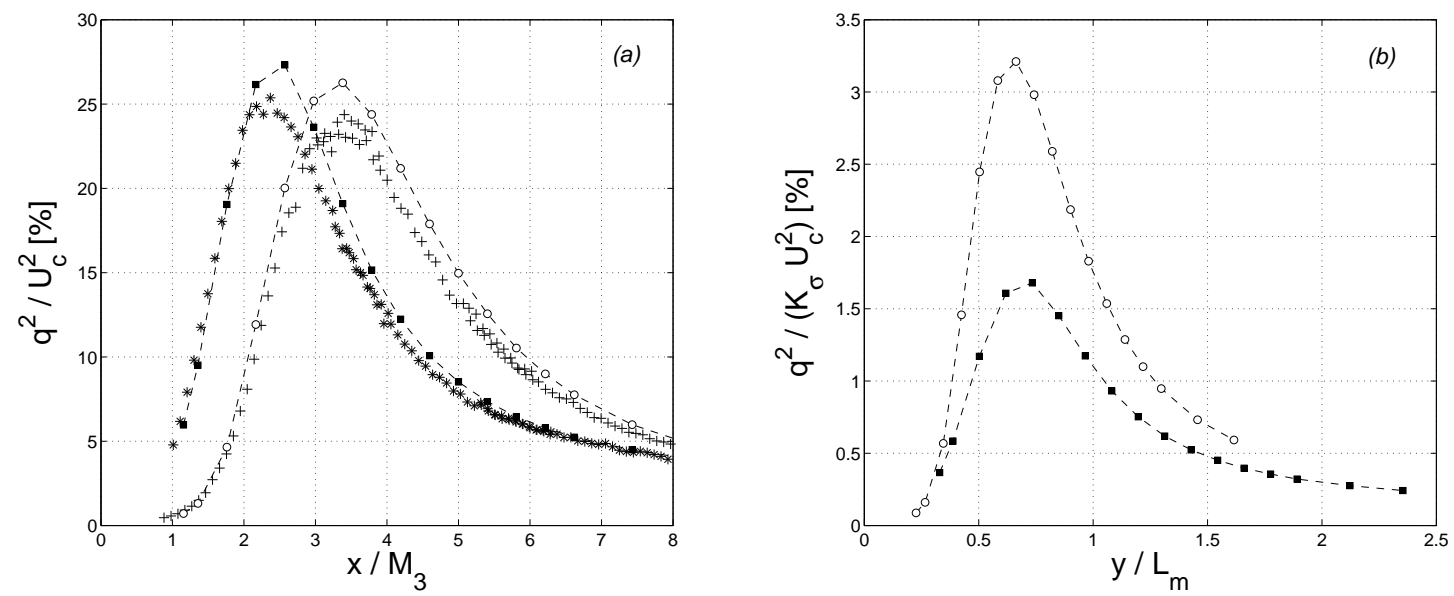

Figure 12: (a) Streamwise evolution of the dimensionless centreline turbulent kinetic energy $q^{2} / U_{c}^{2}$ with respect to the streamwise distance $x$ scaled by the plate's mesh size $M_{3}$. (b) Streamwise evolution of the dimensionless centreline turbulent kinetic energy $q^{2} /\left(K_{\sigma} U_{c}^{2}\right)$ with respect to the streamwise distance $x$ scaled by the merging length $L_{m}$. Symbols:,+ MoSTI injector (PIV measurements); *, MuSTI injector (PIV measurements); $\bigcirc, M o S T I$ injector ( $L D V$ measurements); $\mathbf{\square}, M u S T I$ injector ( $L D V$ measurements). 
For both injectors, Figure 12(a) shows the streamwise variation of this quantity measured from both PIV $\left(q^{2}=\left\langle u^{2}\right\rangle+2\left\langle v^{2}\right\rangle\right)$ and $L D V\left(q^{2}=\left\langle u^{2}\right\rangle+\left\langle v^{2}\right\rangle+\left\langle w^{2}\right\rangle\right)$. We point out that although the experimental curves obtained from both techniques reasonably agree, the PIV measurements systematically underestimate the turbulent kinetic energy compared to the $L D V$ technique. This difference is explained by the low-pass filtering of PIV system [20]. Small in the near-field of the injectors because of the low turbulence level, the discrepancies between $P I V$ and $L D V$ slightly decrease far away from the plate due to the increase of the Kolmogorov scale $\eta$ resulting into a better PIV resolution (see next section).

For both injectors, the turbulent kinetic energy builds up in the vicinity of the latest plate until a maximum and then decays monotonically. Due to transverse exchange enhancement, the evolution of the turbulent kinetic energy $q^{2}$ is much faster for the MuSTI device than for the MoSTI injector. This is evidenced by the location of the peak of energy which appears much closer to the injector for the MuSTI device by about 35\%. Figure 12(b) evidences the strong dependence between the energy peak location and the merging length $L_{m}$ meaning that $q^{2}$ decays as soon as the jets issuing from the plates interact. Moreover, this plot shows that, relatively to the total pressure loss imposed by the injectors, the turbulent kinetic energy generated by the MuSTI decays much more slowly than that of the MoSTI. This is a direct consequence of the acceleration of the transverse exchanges due to the multi-scale injection.

Derived from Navier-Stokes equations [25], the governing equation of turbulent kinetic energy $q^{2}$ can be expressed, in cylindrical-coordinates, as follows:

$$
\begin{array}{r}
-\underbrace{U \frac{\partial q^{2}}{\partial x}-V \frac{\partial q^{2}}{\partial y}}_{\text {Convection }}-\underbrace{\frac{\partial\left\langle u q^{2}\right\rangle}{\partial x}-\frac{1}{y} \frac{\partial y\left\langle v q^{2}\right\rangle}{\partial y}}_{\text {Diffusion }} \\
-\underbrace{2\left[\left\langle u^{2}\right\rangle \frac{\partial U}{\partial x}+\left\langle v^{2}\right\rangle \frac{\partial V}{\partial y}+\langle u v\rangle \frac{\partial U}{\partial y}\right]}_{\text {Production }} \\
+P-2 \epsilon=0
\end{array}
$$

where $P$ represents the turbulent kinetic energy transport by pressure and $\epsilon$ is the turbulent kinetic energy dissipation rate per mass unit. The first three terms (Convection, Diffusion and Production) of Equation (11) can be easily evaluated from PIV measurements. The pressure term $P$ is usually unaccessible by a direct evaluation and is commonly deduced from the budget of Equation (11). Unfortunately, low-pass filtering inherent to PIV system avoids the accurate estimation of the dissipation $\epsilon$ (see e.g. [20], [26]). The spectral corrections recently given by Lavoie \& al. [26] are restricted to homogeneous and isotropic turbulence which is obviously not representative of the injector near-field. Using the continuity equation and dropping off small terms, Equation (11) simplifies to the following form on the tunnel's centreline:

$$
-U_{c} \frac{\partial q^{2}}{\partial x}-\frac{1}{y} \frac{\partial y\left\langle v q^{2}\right\rangle}{\partial y}-2\left(\left\langle u^{2}\right\rangle-\left\langle v^{2}\right\rangle\right) \frac{\partial U_{c}}{\partial x}+P-2 \epsilon=0 .
$$

The different terms involved in Equation (12) are plotted in Figures 13(a), 13(b), 13(c) and 13(d) with respect to the dimensionless distance $x / L_{m}$ for both injectors. The dissipation $\epsilon$ is estimated by two independent ways:

$$
\epsilon_{q}=- \text { Convection }- \text { Diffusion }- \text { Production, }
$$


and

$$
\epsilon_{h}=3 \nu\left[\left\langle\left(\frac{\partial u}{\partial x}\right)^{2}\right\rangle+\left\langle\left(\frac{\partial v}{\partial x}\right)^{2}\right\rangle+\left\langle\left(\frac{\partial w}{\partial x}\right)^{2}\right\rangle\right],
$$

where gradients are inferred from $L D V$ measurements and Taylor's hypothesis.
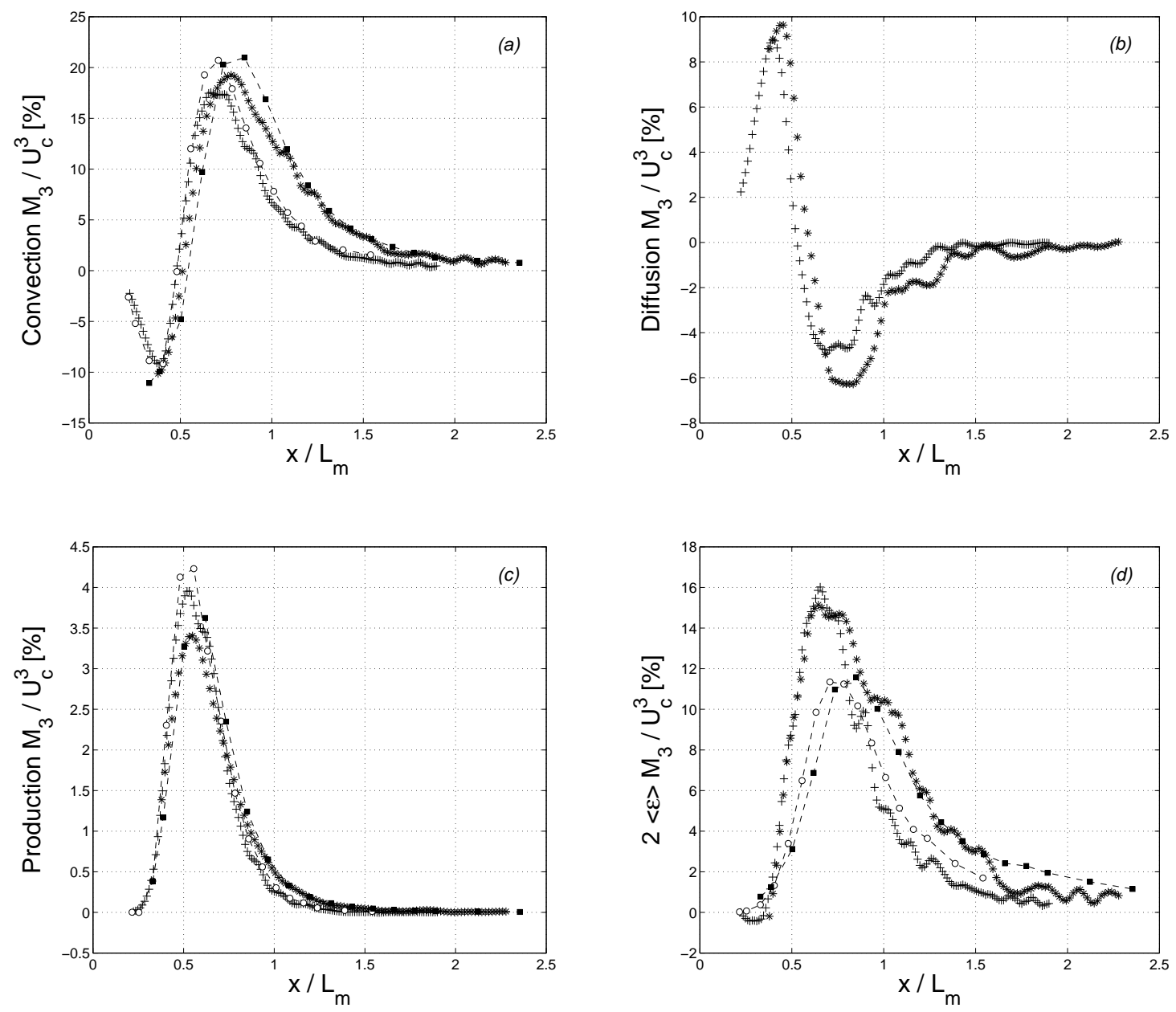

Figure 13: Streamwise evolution of the terms appearing in the budget equation of the turbulent kinetic energy: Convection (a), Diffusion (b), Production (c) and Dissipation (d) with respect to the normalized streamwise distance $x / L_{m}$ for both injectors. Symbols: +, MoSTI injector (PIV measurements); *, $M u S T I$ injector (PIV measurements); $\bigcirc, M o S T I$ injector ( $L D V$ measurements);, MuSTI injector ( $L D V$ measurements).

Equation (13) evaluates dissipation through large-scale properties $(P I V)$, while Equation (14) is a more direct evaluation through small-scale gradients $(L D V)$ for which homogeneity is assumed [27]. The estimation of $\epsilon_{q}$ requires the pressure-related term $P$ to be negligible. This assumption can be checked by comparing both $\epsilon_{q}$ and $\epsilon_{h}$. Results plotted in Figure 13(d) show that this assumption is fairly well fulfilled beyond $x / L_{m}>1$, i.e. in the nearly homogeneous and isotropic region.

Although the various terms of the budget equation evolve in a very similar way for both injectors, some differences remain, especially beyond $x / L_{m}=0.5$. Upstream this position, the lateral diffusion 
term almost compensates the axial convection term which contributes negatively to the turbulent kinetic energy budget meaning that the mean flow convects energy from low energy regions towards high energy regions. On the contrary, the lateral energy diffusion brings energy produced in the lateral shear layer towards the centreline.

Beyond $x / L_{m}=0.5$, convection and diffusion terms change sign. This position coincide with the end of the potential core $\left(\delta / D_{3} \sim 1\right)$ resulting into a peak of turbulent kinetic energy production which is compensated by the negative lateral diffusion term. Then, these two terms decrease in magnitude to become negligible further downstream implying [12]:

$$
-U_{c} \frac{\partial q^{2}}{\partial x}-2 \epsilon=0
$$

This equation reflects that in decaying turbulence, the convection term is compensated by the dissipation. In this region, all terms computed for the MuSTI injectors are higher in magnitude than those of the MoSTI device.

The results obtained from the study of the turbulent kinetic energy lead to conclusions:

(i) the turbulent energy generated by the multi-scale injection is more intense partly due to an higher pressure loss.

(ii) the nearly homogeneous and isotropic region is characterised by a very large turbulence intensity $\left(\sqrt{q^{2} / 3} / U_{c} \approx 15 \%\right)$ compared to standard grid-generated turbulence $(\approx 3 \%)$.

\subsection{Characteristic length-scales and two-point kinetic energy budget}

We remind that the main goal of the present work is to develop a new kind of turbulence injector enable to quickly inject energy over a broad range of scales. The implicit idea is to act onto energy transfer by modifying the energy's cascade. The latter is characterised by both the integral lengthscale $\Lambda_{u}$ (computed from the auto-covariance of streamwise fluctuation $u$ ) and the Kolmogorov scale $\eta$ $\left(\equiv\left(\nu^{3} / \epsilon_{h}\right)^{1 / 4}\right)$ which are plotted in dimensionless form in Figures 14(a) and 14(b) for both injectors on the tunnel's centreline. In the nearly homogeneous and isotropic region, $\Lambda_{u}$ and $\eta$ increase with respect to $x$. For both injectors, the integral length-scale $\Lambda_{u}$ is controlled by the largest mesh size $M_{3}$ which can be interpreted as the spanwise interaction distance. However, the integral length-scale $\Lambda_{u}$ generated by the MuSTI injector is significantly smaller than that of the MoSTI injector. This observation applies also to the Kolmogorov scale.

Although the turbulent scales generated by the MuSTI device are systematically smaller than those of the MoSTI injector, the Taylor-based Reynolds number $\operatorname{Re}_{\lambda}\left(\equiv \sqrt{q^{2} / 3} \lambda / \nu\right.$ with $\lambda=\sqrt{5 \nu q^{2} / \epsilon_{h}}$ the Taylor micro-scale) of the MUSTI injector is higher (by about 30\%) than that of the MoSTI injector as shown in Figure 15. This is due to the acceleration of cascade exchange allowing to reach isotropic region with much larger turbulence intensity which compensates the decrease in turbulent length-scales. Although the values of $R e_{\lambda}$ reported here remain moderate $(\approx 80)$, one has to keep in mind that the experimental wind tunnel is quite small in comparison with usual facilities (see e.g. [12] for comparison).

The way the turbulent energy is injected by both devices can be investigated with structure functions which represent the energy of a scale. These are defined by using spatial increments, i.e. velocity differences between two space points separated by a vector $r$ :

$$
\Delta u_{i}(\boldsymbol{r})=u_{i}(\boldsymbol{x}+\boldsymbol{r})-u_{i}(\boldsymbol{r})
$$

where $i$ designates any velocity component. Under the isotropy hypothesis, the scalar $S_{2 q}=$ $(\Delta q)^{2}(\boldsymbol{r}) \equiv\left(\Delta u_{i}\right)^{2}(\boldsymbol{r})$ only depends on $r$, the modulus of the separation $\boldsymbol{r}$, and represents the total kinetic energy of the scale $r$. These increments are calculated using LDV data, and the separation 

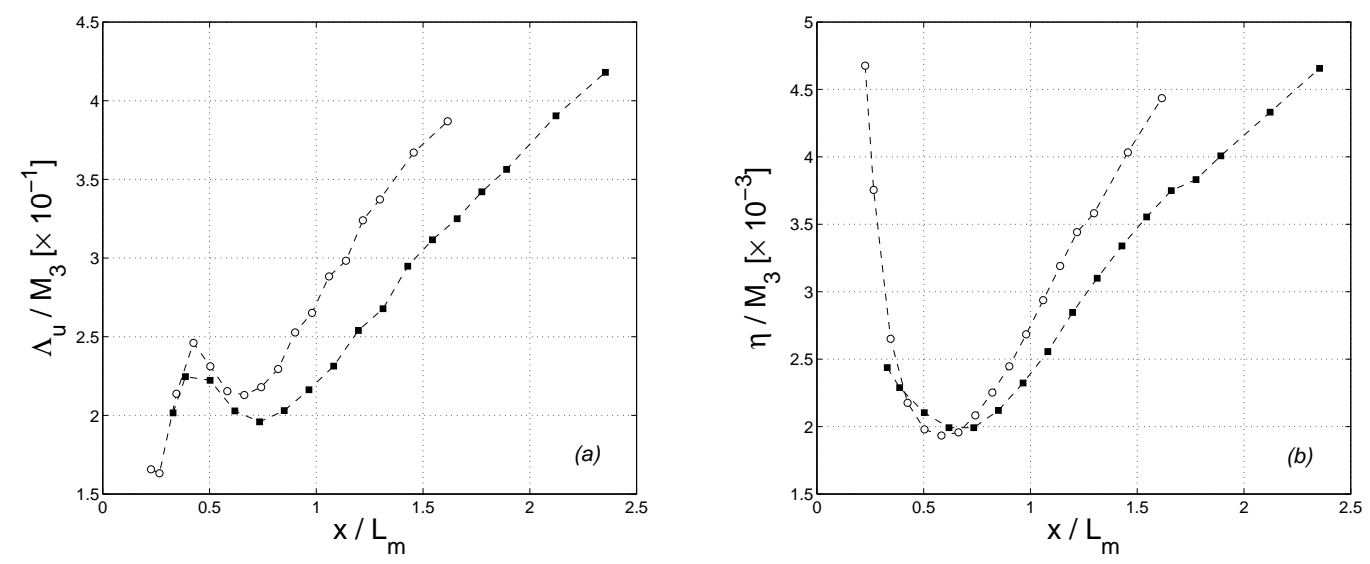

Figure 14: Streamwise variation of the normalized integral length-scale $\Lambda_{u} / M_{3}$ (a) and Kolmogorov scale $\eta / M_{3}(b)$. Symbols: $\bigcirc$, MoSTI injector; $\mathbf{\square}, M u S T I$ injector.

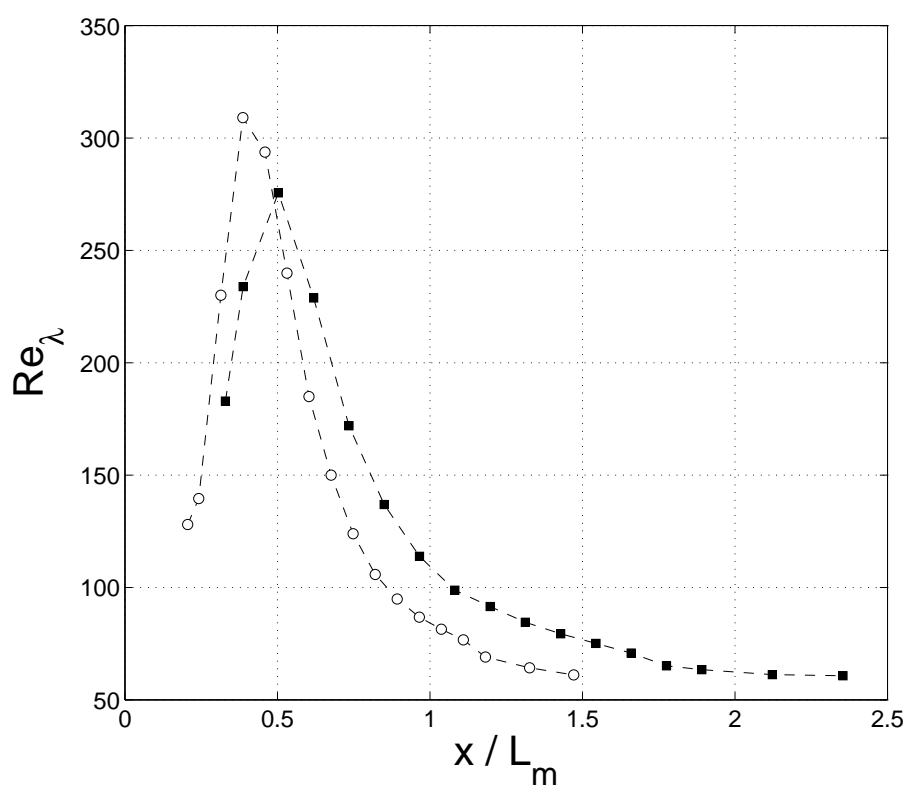

Figure 15: Taylor-based Reynolds number evolution. Symbols: $\bigcirc$, MoSTI injector;, MuSTI injector. 
$r$ is obtained from the temporal lag, via the Taylor's hypothesis (the local Taylor's hypothesis has also been used, with no significant difference on the results). An important remark is to be done here. The structure functions calculation from data non-uniformly sampled in time ( $L D V$ technique) does not require an equidistant time-resampling (as it is the case for calculating spectra) unlike Fourier transform.

In the context of the present work, it is of particular relevance to compare $S_{2 q}$ for both devices, see Figure $16(\mathrm{a})$, at the same spatial location (here $x / M_{3} \approx 2$ ). The representation is done as a function of $r / \eta$, where $\eta$ is the Kolmogorov microscale.
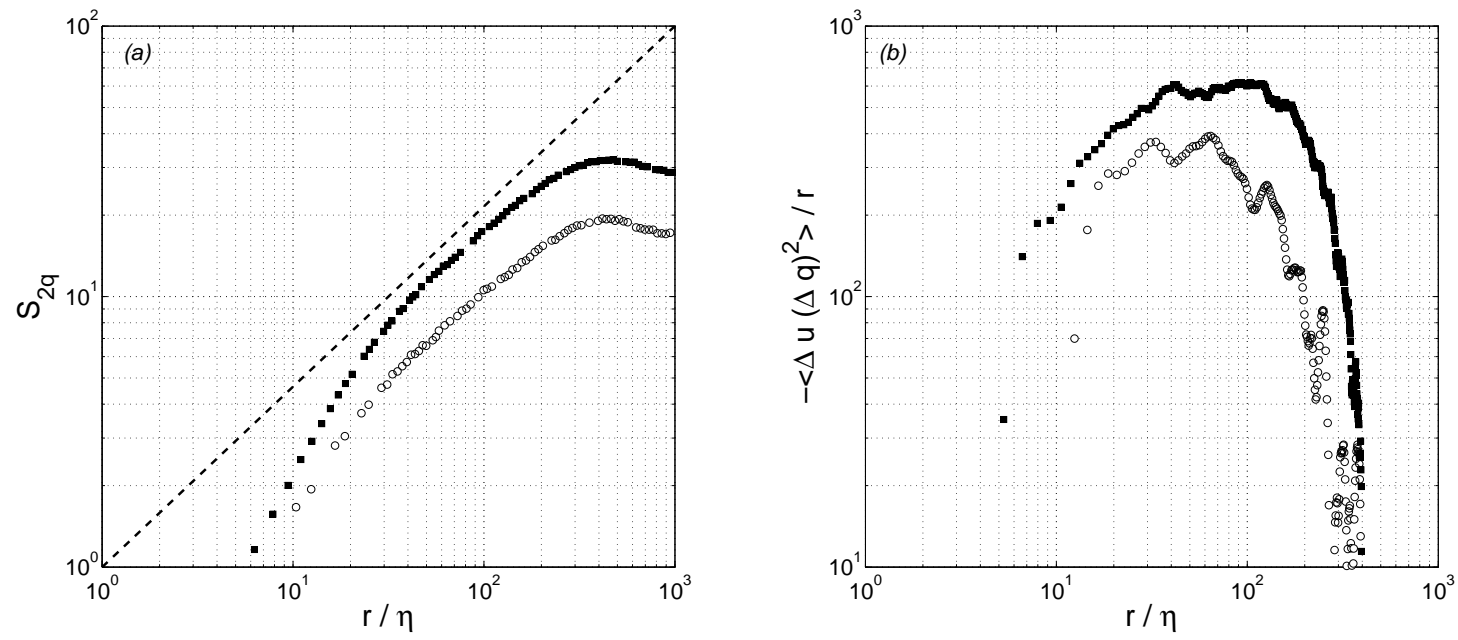

Figure 16: (a) Second-order structure functions $\left\langle(\Delta q)^{2}\right\rangle$. The black dotted line represents the $r^{2 / 3}$ scaling. (b) Third-order structure functions divided by the separation $r,-\left\langle\Delta u(\Delta q)^{2}\right\rangle / r$. Symbols: $\bigcirc$, MoSTI injector; $\mathbf{\square}, M U S T I$ injector.

The first thing to be noted is that the MuSTI device presents (approximately twice) higher values of $S_{2 q}$ than those of MoSTI, and this holds for all the range of scales. The large-scale limit $(r \rightarrow \infty)$ of $S_{2 q}$ is (under the hypothesis of homogeneity) twice the total kinetic energy $q^{2}$. The ratio of the large scale limits $(\approx 1.8)$ fully corresponds to the ratio of the total kinetic energies of both devices. For both flows, noteworthy are the energy accumulations at large scales, approximately peaking at the scale $400 \eta$, corresponding to the injector(s) mesh $M_{3}$. A further analysis of $S_{2 q}$ reveals that both of them exhibit a restricted scaling range (RSR), for which the scaling is very nearly equal to the asymptotic value of $2 / 3$ (equivalent to the $-5 / 3$ exponent in the spectral space).

A deeper insight into the nature of the turbulent cascade, albeit in the context of local isotropy, is provided by the third-order structure function $-\left\langle\Delta u(\Delta q)^{2}\right\rangle$, where the velocity component $u$ is parallel to the spatial separation $\boldsymbol{r}$, taken along the mean flow direction. The mathematical relation between the second-and third-order structure functions, obtained under the assumption of locally isotropic, high-Reynolds number turbulence, is e.g. [28],

$$
-\left\langle\Delta u(\Delta q)^{2}\right\rangle+2 \nu \frac{d}{d r}\left\langle(\Delta q)^{2}\right\rangle=\frac{4}{3} \epsilon r .
$$

For very small scales, it complies with $\epsilon_{h}$. Equation (17) signifies that the energy transferred at a scale $r$ is (only) done through turbulent advection (the third-order term) and molecular diffusion.

Figure 16(b) represents term $-\left\langle\Delta u(\Delta q)^{2}\right\rangle / r$, for both devices. This terms should be equal to the constant value of $4 / 3 \epsilon$, for high enough Reynolds numbers. For the MoSTI device, a very restricted 
plateau is present for a range of scales lying between 30-60 Kolmogorov scales. On the contrary, the flow associated to the $M u S T I$ device exhibits broadened turbulent energy transfer, since term $-\left\langle\Delta u(\Delta q)^{2}\right\rangle / r$ is characterised by a plateau much better defined over a range of scales $40-150$ Kolmogorov scales. This difference cannot be attributed to a Reynolds number effect because $R e_{\lambda}$ is roughly the same $(\approx 220)$ for both devices at this location. This result proves that energy transfer through turbulence is, at the same spatial position, enhanced by using the MuSTI injector.

As earlier emphasized, Equation (17) is only valid for high Reynolds numbers, and for a limited range of scales. For moderate Reynolds numbers associated to real flows, it is therefore of interest to study the scale-by-scale energy transport, by also taking into account large-scale effects (turbulent diffusion, decay, production). The final equation, also obtained in the context of local homogeneity (pressure-containing terms are neglected) and local isotropy, writes [29], [30]

$$
\begin{array}{r}
-\left\langle\Delta u(\Delta q)^{2}\right\rangle+2 \nu \frac{d}{d r}\left\langle(\Delta q)^{2}\right\rangle-\frac{U}{r^{2}} \int_{0}^{r} s^{2} \frac{\partial}{\partial x}\left\langle(\Delta q)^{2}\right\rangle d s \\
-\frac{1}{r^{2}} \int_{0}^{r} s^{2}\left[\frac{1}{2}\left(\frac{\partial}{\partial x_{\alpha}}+\frac{\partial}{\partial x_{\alpha}^{+}}\left\langle\left(u_{\alpha}+u_{\alpha}^{+}\right)(\Delta q)^{2}\right\rangle\right)\right] d s \\
-2 \frac{\partial U}{\partial x} \frac{1}{r^{2}} \int_{0}^{r} s^{2}\left(\left\langle(\Delta u)^{2}\right\rangle-\left\langle(\Delta v)^{2}\right\rangle\right) d s=\frac{4}{3} \epsilon r
\end{array}
$$

where $s$ is a dummy variable and repeated indices indicate summation. This equation could be written, after dividing by $\epsilon r$, in the following dimensionless form

$$
A^{*}+B^{*}+D^{*}+T D^{*}+P R^{*}=C^{*}
$$

where $C^{*}=4 / 3, A^{*}$ is the term associated to turbulent transfer, $B^{*}$ to molecular effects, $D^{*}$ is the inhomogeneous ('decay') term along the streamwise direction $x, T D^{*}$ is the turbulent diffusion and $P R^{*}$ is the production term.

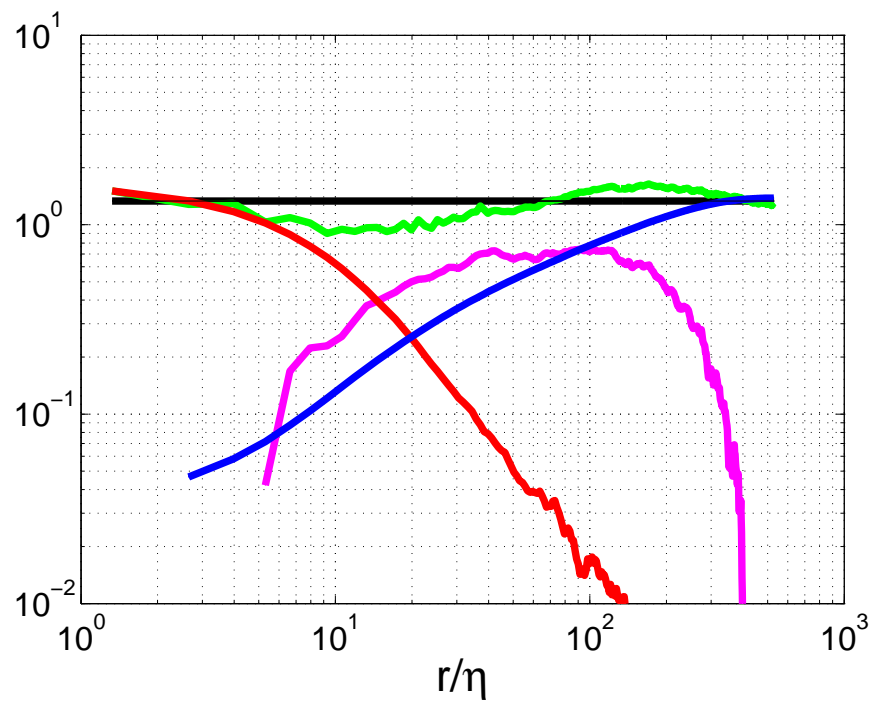

Figure 17: Scale-by-scale kinetic energy budget for the MuSTI device. Term $A^{*}$ (magenta); Term $B^{*}$ (red); Term $D^{*}$ (blue); Term $A^{*}+B^{*}+D^{*}$ (green). The black solid line represents the value of $4 / 3$.

It is straightforward to show that, in the context of homogeneous turbulence, the large-scale limit of Equation (18) is Equation (12). At the streamwise position investigated here, and as previously 
emphasized, the effect of turbulent diffusion compensates that of turbulent production, and therefore the 1-point energy budget equation reduces to Equation (15), in which only the decay effect has to be taken into account. In this context, we consider that $T D^{*}+P R^{*} \approx 0$, which leads to only consider the decay effect, as it is the case in decaying grid turbulence.

Terms $A^{*}, B^{*}, D^{*}$ and $C^{*}$ are calculated for the MuSTI injector, at the position $x / L_{m}=0.6$, where the total kinetic energy is maximum, and after which the energy decay is representative of the flow. These terms are represented in Figure 17, as functions of $r / \eta$. Term $B^{*}$, representing energy transferred via molecular effects, is only present at small scales, and is negligible for large scales. Note also that for the dissipative range domain, term $B^{*}$ equilibrates $C^{*}=4 / 3$, in agreement with the definition of $\epsilon_{h}$. Term $A^{*}$ is important for an intermediate range of scales (RSR), and negligible for both very small and very large scales. At this Reynolds number $\left(R e_{\lambda} \approx 220\right)$, term $A^{*}$ does not balance $C^{*}$, signifying that the energy transfer is not only performed by turbulent advection. Large-scale (here, decay) effect represented by term $D^{*}$ is increasingly important for larger and larger scales. It equilibrates term $A^{*}$ in the middle of the RSR, and it is significantly larger for scales equal and larger than the integral scale. It equilibrates by itself term $C^{*}$ for the largest scales of the flow, in agreement with Equation (12). Finally, $A^{*}+B^{*}+D^{*}$ (green curve) equilibrates term $C^{*}$ reasonably well over the whole range of scales, with smaller values (20\% lower) for the RSR scales, and larger (10\%) for the largest scales of the RSR. This disagreement is attributable to the effect of turbulent diffusion and production (not considered here) and also to departures from local isotropy.

We conclude here on the cold flow configuration saying that

(i) the large-scales of the flow remain controlled by the largest plate, i.e. by the spanwise interaction distance.

(ii) the small-scales are intensified in terms of energy and their range is broadened by the multi-scale injection thanks to the enhancement of the energy transfer term.

iii) the MuSTI device roughly behaves as a grid turbulence starting with $x / L_{m}=0.6$ position, for which all the shear layers are well mixed, global and local isotropy hold well, and the energy production is swept by turbulent diffusion. As far as the MoSTI device is concerned, the same analysis (developed in the context of locally isotropic turbulence), does not hold as well and therefore is not reported here.

The issue we address in the following is the influence of the multi-scale energy injection mode onto the interaction between combustion and intense homogeneous and isotropic turbulence.

\subsection{Interaction with premixed combustion}

The following part is dedicated to the qualitative and quantitative description of the interaction between the turbulence generated by both injectors and premixed combustion. For this purpose, an air/methane mixture is used as working fluid with an equivalence ratio $\phi=0.6$. A V-shaped flame is attached on a tiny heated rod located in the homogeneous and isotropic region of the flow determined from the investigation in non-reactive configuration. The location $x_{\text {rod }}$ of the heated rod is given in Table 3 as well as the main flow properties of the turbulent flow at the rod position.

Relatively to the jet interaction origin, the heated rod is almost located at the same position $x_{\text {rod }} / L_{m}$ for both turbulence generators. At this location, the turbulent flow generated by both injectors is nearly homogeneous and isotropic with comparable turbulence intensity level. However, the MuSTI injector generates slightly smaller turbulent scales which are more energetic than those of the MoSTI device. This property improves the ability of multi-scale generated turbulence to interact with the flame at both 


\begin{tabular}{cccccccccc}
\hline Injector & $\frac{x_{\text {rod }}}{L_{m}}$ & $H$ & $I$ & $\frac{\sqrt{q^{2} / 3}}{U_{c}}(\%)$ & $\sqrt{q^{2} / 3}(\mathrm{~m} / \mathrm{s})$ & $\Lambda_{u}(m m)$ & $\lambda(m m)$ & $\eta(m m)$ & $R_{\lambda}$ \\
\hline \hline MoSTI & 1.45 & 0.03 & 1.18 & 14 & 0.58 & 9.1 & 1.5 & 0.10 & 64 \\
MuSTI & 1.34 & 0.04 & 1.14 & 18 & 0.88 & 6.6 & 1.3 & 0.08 & 85 \\
\hline
\end{tabular}

Table 3: Main properties of the turbulent flow at the location of the heated rod.

large- and small-scales as evidenced by the examples of binary flame images obtained downstream both injectors (see Figure 18). Although the turbulence statistics are comparable for both configurations, one can notice an evident difference in the global shape of the flames. The wrinkling of the front flame edges is clearly much larger for the MuSTI injector as testified by the turbulent flame brush and the local extinctions allowing unburnt gases pockets to penetrate deeply inside the burnt gases. Such phenomena are not visible for the MoSTI injector.

These differences partly result from the amplification of the turbulence intensity as reported in previous works (e.g. [31], 32]). Combining this effect with the reduction of turbulent length-scales can also affect significantly the flame stretch [33] by acting of both strain rate and flame curvature.

The competition between the chemical reaction rate and the turbulence is characterised by:

1. the ratio between the integral time-scale $\tau_{t}=\Lambda_{u} / \sqrt{q^{2} / 3}$ and the chemical time-scale $\tau_{c}$ for the large-scale turbulence.

2. the ratio between the chemical time-scale $\tau_{c}$ and the Kolmogorov time $\tau_{\eta}=\eta / u_{\eta}$ (with $u_{\eta}=$ $(\nu \epsilon)^{1 / 4}$ the Kolmogorov velocity) for the small-scale turbulence .

The chemical time-scale $\tau_{c}$ is defined as the ratio between the laminar flame thickness $\delta_{L}^{0}$ (estimated from the maximal temperature gradient $\delta_{L}^{0}=\frac{T_{b}-T_{u}}{(\nabla T)_{\max }}$ with $T_{u}$ the temperature in fresh gases and $T_{b}$ the temperature of burnt gases) and the laminar flame speed $S_{L}^{0}$ [34]. These parameters are reported in Table 4 for our methane/air mixture with an equivalence ratio $\phi=0.6$. The large-scale dynamic ratio can be expressed via Damköhler number

$$
D a=\frac{\Lambda_{u}}{\delta_{L}^{0}} \frac{S_{L}^{0}}{\sqrt{q^{2} / 3}}
$$

while the small-scale dynamic ratio is represented by the Karlovitz number

$$
K a=\left(\frac{\sqrt{q^{2} / 3}}{S_{L}^{0}}\right)^{3 / 2}\left(\frac{\Lambda_{u}}{\delta_{L}^{0}}\right)^{-1 / 2}
$$

These numbers are given in Table 4 for both injectors.

\begin{tabular}{cccccc}
\hline Injector & $\phi$ & $S_{L}^{0}(\mathrm{~m} / \mathrm{s})^{a}$ & $\delta_{L}^{0}(\mathrm{~mm})^{b}$ & $K a$ & $D a$ \\
\hline \hline MoSTI & 0.6 & 0.11 & 1.02 & 4.09 & 1.68 \\
MuSTI & 0.6 & 0.11 & 1.02 & 8.82 & 0.81 \\
\hline
\end{tabular}

Table 4: Main chemical properties of the fuel-air mixture. ${ }^{a}$ estimated from [35]. ${ }^{b}$ estimated from [36].

The MUSTI device shortens both large- and small-scale turbulent times compared to the chemical time as testified by the decrease of Damköhler number $D a$ and the increase of Karlovitz number $K a$ (by a factor $\approx 2$ ). 

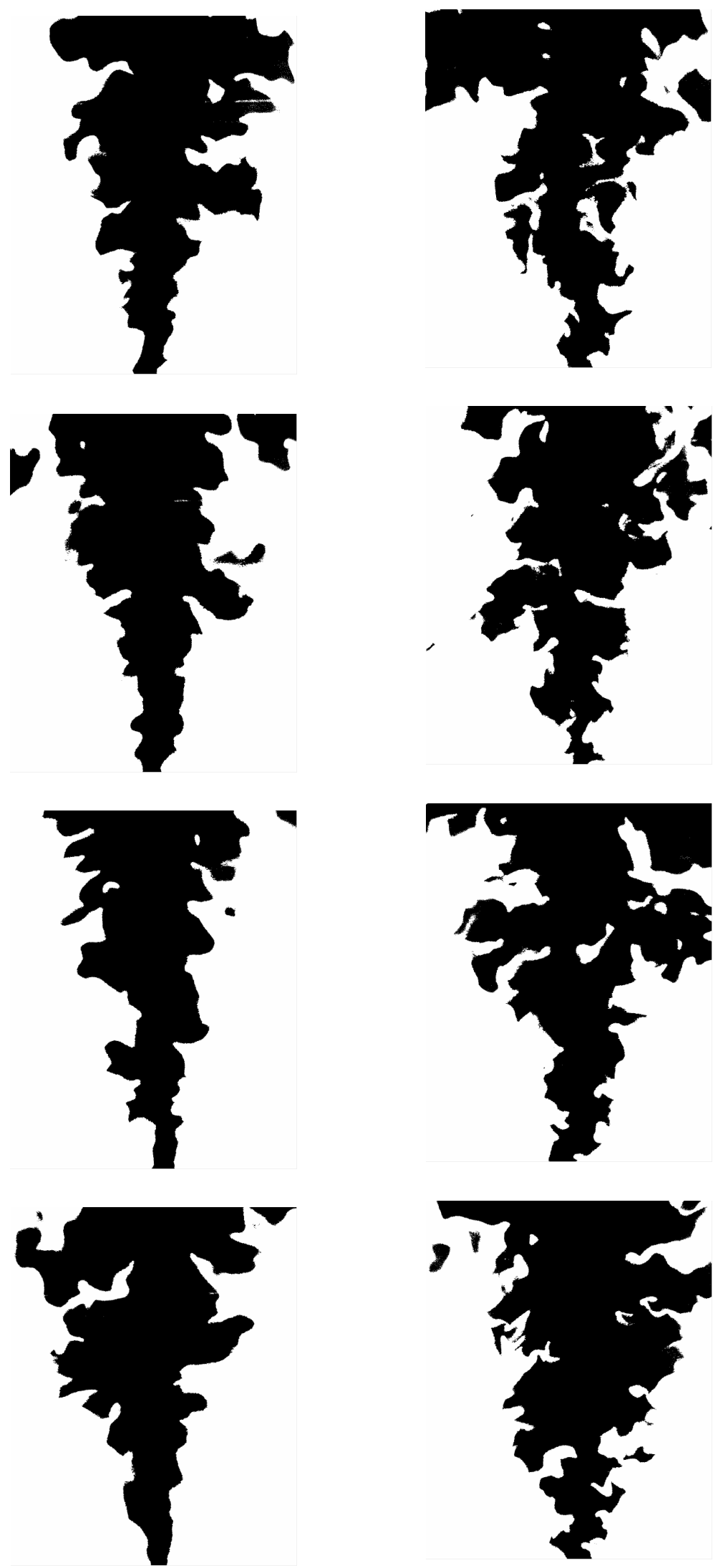

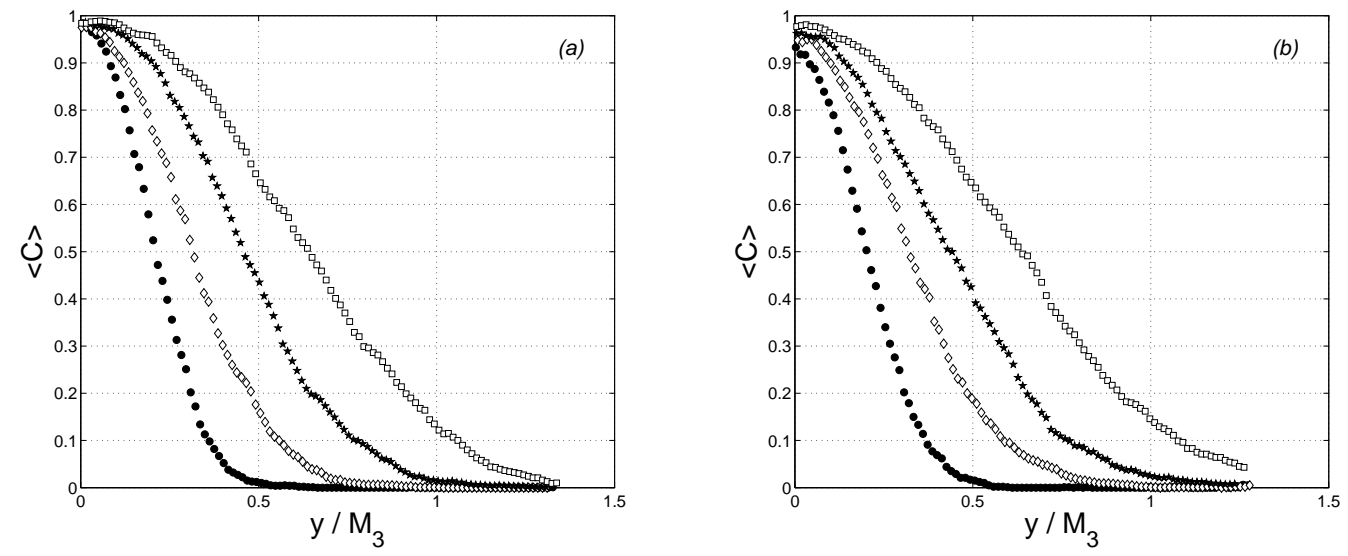

Figure 19: Transverse profiles of the mean progress variable $\langle C\rangle$ at various distances from the heated rod downstream the MoSTI injector (a) and the MuSTI injector (b). Symbols: $\bullet,\left(x-x_{\text {rod }}\right) / M_{3}=1$; $\diamond,\left(x-x_{\text {rod }}\right) / M_{3}=1.5 ; \star,\left(x-x_{\text {rod }}\right) / M_{3}=2 ; \square,\left(x-x_{\text {rod }}\right) / M_{3}=2.5 ;$

One of the main consequences of the turbulent dynamics amplification by multi-scale injection mode is to increase the flame wrinkling as illustrated in Figure 18. This phenomenon can be quantified by the flame brush thickness $\delta_{t}$ which is evaluated from the profile of the Reynolds average progress variable $\langle C\rangle$. This mean progress variable distribution corresponds to a map of flame front presence probability. It is obtained from instantaneous binarized images $(C=0$ in fresh gases and $C=1$ in burnt gases), representing the instantaneous map of the progress variable. It is important to notice that the spatial resolution of the tomography technique is not good enough to investigate the local flame structure. We restrict therefore the discussion on the global properties of the flame, i.e. at large-scale. In this way, we implicitly assume that the flame front is thin with respect to the spatial resolution of the measurements.

In Figures 19(a) and 19(b), we report the profiles of the mean progress variable $\langle C\rangle$ measured at several distances from the heated rod for the MoSTI and the MuSTI injectors respectively. These figures evidence the flame expansion with the distance from the heated rod. For the MuSTI injector, one can remark that the maximum of the mean progress variable $\langle C\rangle_{\max }$ remains lower than unity and tends towards 1 with increasing streamwise distance. This behaviour cannot be attributed to measurements uncertainties but is related to the strong wrinkling induced by the multi-scale forcing.

The flame brush thickness $\delta_{t}$ has been computed from the mean progress variable $\langle C\rangle$ profiles according to [37]:

$$
\frac{1}{\delta_{t}}=\left(\frac{\partial\langle C\rangle}{\partial y}\right)_{\max }
$$

In the case of the MuSTI injector, the mean progress variable profiles have been normalized by $\langle C\rangle_{\max }$ as suggested by Lipatnikov \& Chomiak [38]. The flame brush thickness $\delta_{t}$ scaled by the integral scale $\Lambda_{u}$ is plotted against the dimensionless distance from the flame-holder $\frac{x-x_{\text {rod }}}{M_{3}}$ in Figure 20 (a) for both injectors. As expected, the flame brush thickness $\delta_{t}$ increases versus $x$. Moreover, the flame brush thickness $\delta_{t}$ is increased by about $30 \%$ for the $M u S T I$ injector confirming the amplification of the flame wrinkling compared to the MoSTI device.

The growth of the flame brush thickness $\delta_{t}$ can be described by the Taylor's turbulent diffusion approach (see e.g. the review article from Lipatnikov \& Chomiak [38]) expressed as: 

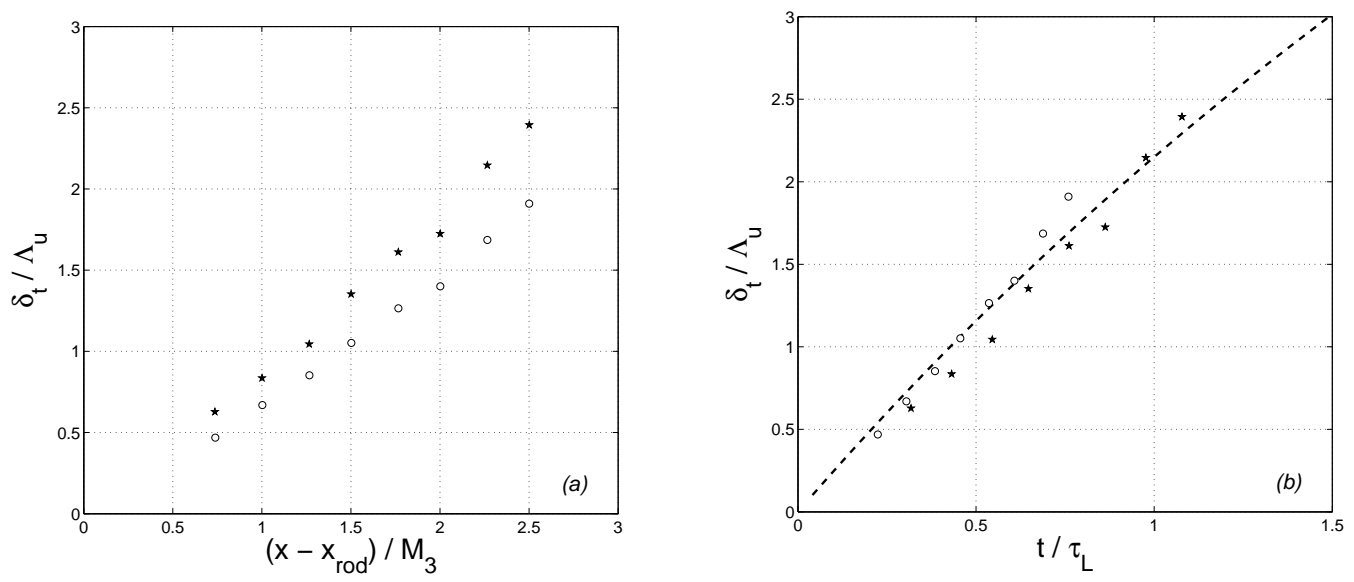

Figure 20: (a) Streamwise variation of the normalized flame brush thickness $\delta_{t} / \Lambda_{u}$ vs. the dimensionless distance from the heated rod $\frac{x-x_{\text {rod }}}{M_{3}}$. (b) Comparison with the Taylor's turbulent diffusion law (dashed line) given in equation 23. Symbols: o, MoSTI injector; *, MuSTI injector.

$$
\delta_{t}=\sqrt{2 \pi} \Lambda_{u}\left\{2\left(\frac{t}{\tau_{L}}\right)\left[1-\left(\frac{\tau_{L}}{t}\right)\left(1-e^{-t / \tau_{L}}\right)\right]\right\}^{1 / 2}
$$

where $t$ is the elapsed time from the source (heated rod) and $\tau_{L}=\frac{\Lambda_{u}}{u^{\prime}}$ (with $u^{\prime}$ the axial rootmean square velocity) [39] is the Lagrangian integral time-scale. The comparison between the flame brush thickness $\delta_{t}$ evolution and the Taylor's turbulent diffusion law is given in Figure 20(b) for both injectors. Experimental data fairly well collapse on diffusion law of Equation (23) indicating that the flame brush is mainly controlled by the large-scale turbulence surrounding the flame. Our results are in good agreement with those reported by Goix \& al. [40] and Renou \& al. [32] in grid-generated turbulence for various flammable mixtures.

We conclude this section by presenting the expected area covered by the flame generated downstream the MoSTI injector and the MuSTI device in Figure 21. The reported point are computed from the turbulence parameters evaluated at the position $x_{\text {rod }}$ (see Table 3) and the chemical properties of the mixture we have used (see Table 4). For comparison, experimental results obtained by Dunn \& al. [7] for a piloted jet burner, O'Young \& Bilger [5] for bluff-boby and Dinkelacker \& al. [6] for swirl flow are also reported. The flame generated downstream the MoSTI is expected to be in the distributed region, whilst that of the $M U S T I$ device is expected to reach the well-stirred reactor region. The latter is only accessible to highly turbulent flows such as jets [7] or bluff-body [5] turbulence which are flows where homogeneity and isotropy assumptions are far to be fulfilled unlike the turbulent flow generated by the MuSTI injector. However, a straightforward interpretation of these results might be risky. Indeed, combustion diagrams are mainly based on dimensional phenomenology as well as empirical considerations. This means that the frontiers between premixed flame regimes are not so obvious due to the complex interactions between combustion and turbulence.

In the frame of the present study, we cannot reveal the real impact of the multi-scale injection onto the local flame front properties although the flame structure seems to be largely influenced by the MuSTI device. One might expect that the latter, by reducing and reinforcing the turbulent small-scales, would enable them to compete against heat release which enhances dissipation via viscous effects [9]. This process might favour the penetration of the turbulent small-scales into the flame leading therefore to the thickening of the pre-heated zone. In future, we aim to extend the present work on resolved 


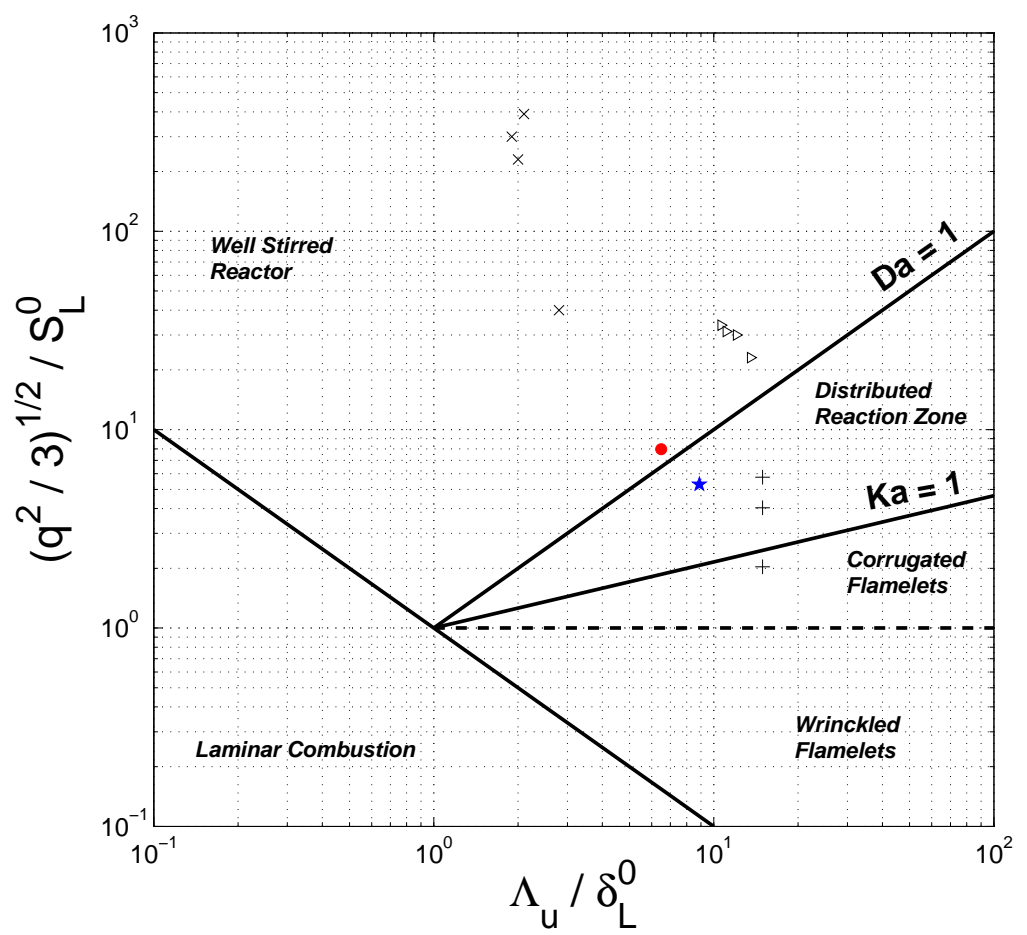

Figure 21: Location of the expected flames in the combustion diagram. Present work : $(\bullet)$ MuSTI

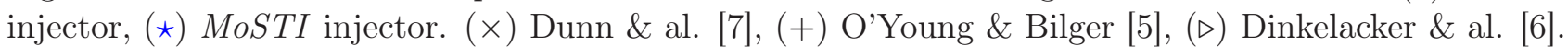

investigation of the flame front by using Rayleigh scattering technique [36] in order to check the influence of the multi-scale injection onto the flame thickness, especially the pre-heated zone.

\section{Conclusions}

The turbulent flow produced by a new kind of turbulence generator, and its interaction with premixed combustion, has been experimentally investigated. This original injector is made of a combination of three perforated plates shifted in space such that both their hole's diameter and their blockage ratio increase with increasing distance. This device enables a delayed multi-scale injection of energy which has been compared with a reference mono-scale injector (individual perforated plate). This qualitative and quantitative comparison has been performed in both a cold and a reactive flow.

\subsection{Main conclusions in the cold flow configuration}

Our results leads to the following observations for the MuSTI injector:

(i) the homogeneous and isotropic region is reached 'earlier', by about 50\%, with isotropy level comparable to standard grid-generated turbulence.

(ii) The merging length $L_{m}$, which corresponds to the jet interaction origin, is found to be the relevant length-scale characterizing the streamwise variation of the turbulent flow.

(iii) The homogeneous and isotropic region is characterised by much larger turbulent intensity $(\approx 15 \%)$ than standard grid-generated turbulence. 
(iv) Due to the multi-scale injection, the turbulent kinetic energy supply is distributed over the whole range of scales (large till the smallest) as emphasized by second-order structure functions.

(v) In the decay region, the turbulent energy transfer at a given scale $r$ is enhanced, as underlined by the third-order structure function $-\left\langle\Delta u(\Delta q)^{2}\right\rangle$.

The scenario we propose for explaining these results is intimately connected to the intrinsic concept of our multi-scale energy injector. Indeed, the turbulence genesis of the multi-scale injector is done gradually, over a much longer distance than the MoSTI (for which, the turbulence injection is only done along the plate thickness). Indeed, for the MuSTI injector, the turbulence birth begins $45 \mathrm{~mm}$ $\left(=3.5 M_{1}+3.5 M_{2}\right)$ upstream the inlet of the wind tunnel. Along this distance the turbulence is gradually reinforced. In connection with the particular energy injection mode (stagnating the flow over a large surface), fluctuations of $u$ along $x$ are principally created in a first time. The fact that this energy supply is done progressively in the flow, the fluctuating velocity $u$ has enough 'time/space' to feed the other two fluctuating velocity components $v$ and $w$. Therefore, the multi-scale injector significantly enhances transverse fluxes accelerating the spatial redistribution of energy, and therefore reaching homogeneity and isotropy more rapidly.

Besides the enhancement of transverse exchanges at large-scale, our work shows that the multiscale injection reinforces the small-scales by accelerating and amplifying the energy transfer. This result might be interpreted as a turbulent cascade by-pass.

\subsection{Main conclusions in the reactive flow configuration}

The interaction of premixed combustion and turbulence generated by multi-scale and mono-scale injector has been qualitatively investigated by front flame visualization. The multi-scale generated turbulence strongly increases the wrinkling of the flame edge provoking local extinctions and propagation of unburnt pockets in the burnt gases. This observation is confirmed by the $30 \%$ increase of the flame brush thickness. The flame produced downstream the multi-scale injector is expected to reach flame regime unaccessible to standard grid-generated turbulence in the combustion diagram. This regime requires highly turbulent level which are usually incompatible with homogeneity and isotropy. The multi-scale injector overcomes these drawbacks by fulfilling the aforementioned conditions. However, due to experimental restrictions, our analysis of flame properties cannot reveal the real influence of the multi-scale injection onto the local flame front. Future works will be specifically dedicated to the investigation of the local flame front, in particular the interaction between the multi-scale generated turbulence and the pre-heated zone.

Acknowledgments: The authors are grateful to Dr. G. Godard for his very useful help with the LDV measurement system, to Dr. F. Corbin for his assistance with the LDV acquisition system. This work has been supported by a Région Haute-Normandie grant.

\section{References}

[1] H. Pitsch, Large Eddy Simulation of turbulent combustion, Ann. Rev. Fluid Mech. 38 (2006), pp. 453-482

[2] N. Peters, Laminar flamelets concepts in turbulent combustion, 21st Symp. on Combustion (1986), pp. $1231-1250$

[3] R. Borghi and M. Champion, Modélisation et théorie des flammes, Technip, Paris (2000) 
[4] T. Poinsot, D. Veynante and S. Candel, Diagrams of premixed turbulent combustion based on direct simulation, 23rd Symp. on Combustion (1990), pp. 613-619

[5] F. O'Young and R.W. Bilger, Scalar gradient and related quantities in turbulent premixed flames, Comb. Flame 109 (1997), pp. 682-700

[6] F. Dinkelacker, A. Soika, D. Most, D. Hofmann, A. Leipertz, W. Polifke, K. Döbbeling, Structure of locally quenched highly turbulent lean premixed flame, Proc. Combust. Inst. 27 (1998), pp. 857-865

[7] M.J. Dunn, A.R. Masri and R.W. Bilger, A new piloted premixed jet to study strong finite-rate chemistry effects, Comb. Flame 151 (2007), pp. 46-60

[8] L. Vervisch, R. Hauguel, P. Domingo and M. Rullaud, Three facets of turbulent combustion modelling: DNS of premixed V-flame, LES of lifted nonpremixed flame and RANS of jet-flame, J. Turbul. 5 (2004)

[9] Y. Nada, M. Tanahashi and T. Miyauchi, Effect of turbulence characteristics on local flame structure of $\mathrm{H}_{2}$-air premixed flames, J. Turbul. 5 (2004)

[10] G.K. Batchelor and A.A. Townsend, Decay of isotropic turbulence in the initial period, Proc. R. Soc. Lond. A 193 (1948), pp. 539-558

[11] S. Corrsin, Turbulence: experimental methods. In Handbuch der Physik, Springer (1963), pp. 524-589

[12] G. Comte-Bellot and S. Corrsin, The use of a contraction to improve isotropy of grid-generated turbulence, J. Fluid Mech. (1966) 25, pp. 657-682

[13] M. Gad-el-Hak and S. Corrsin, Measurements of the nearly isotropic turbulence behind a uniform jet grid, J. Fluid Mech. 62 (1974), pp. 115-143

[14] H. Makita, Realization of a large-scale turbulence field in a small wind tunnel, Fluid Dyn. Res. 8 (1991), pp. $53-64$

[15] L. Mydlarski and Z. Warhaft, On the onset of high-Reynolds-number grid-generated wind tunnel turbulence, J. Fluid Mech. 320 (1996), pp. 331-368

[16] D. Hurst and J.C. Vassilicos, Scalings and decay of fractal-generated turbulence, Phys. Fluids 19 (2007), 035103

[17] J. Tan-Atichat, H.M. Nagib and R.I. Loehrke, Interaction of free-stream turbulence with screens and grids: a balance between turbulent scales, J. Fluid Mech. 114 (1982), pp. 501-528

[18] J. Groth and A.V. Johansson, Turbulence reduction by screens, J. Fluid Mech. 197 (1988), pp. 139-155

[19] O. Dégardin, B. Renou and A.M. Boukhalfa, Simultaneous measurement of temperature and fuel mole fraction using acetone planar induced fluorescence and Rayleigh scattering in stratified flames, Exp. Fluids 40 (2006), pp. 452-463

[20] J.M. Foucault, J. Carlier and M. Stanislas, PIV optimization for the study of turbulent flow using spectral analysis, Meas. Sci. Tech. 15 (2004), pp. 1046-1058

[21] B. Mazzi and J.C. Vassilicos, Fractal-generated turbulence, J. Fluid Mech. 502 (2004), pp. 65-87

[22] E. Villermaux and E.J. Hopfinger, Periodically arranged co-flowing jets, J. Fluid Mech. 263 (1994), pp. 63-92

[23] E.M. Laws and J.L. Livesey, Flow through screens, Ann. Rev. Fluid Mech. 10 (1978), pp. 247-266

[24] H. Tennekes and J.L. Lumley, A first course in turbulence, MIT Press, 1972

[25] S.B. Pope, Turbulent flows, Cambridge University Press, 2000 
[26] P. Lavoie, G. Avallone, F. De Gregorio, G.P. Romano and R.A. Antonia, Spatial resolution of PIV for the measurement of turbulence, Exp. Fluids 43 (2007), pp. 39-51

[27] L. Danaila, F. Anselmet and R.A. Antonia, An overview of the effect of large-scale nonhomogeneities on small-scale turbulence, Phys. Fluids 14 (2002), pp. 2475-2484

[28] R.A. Antonia, M. Ould-Rouis, F. Anselmet and Y. Zhu, Analogy between predictions of Kolmogorov and Yaglom, J. Fluid Mech. 332 (1997), pp. 395-409

[29] L. Danaila, R.A. Antonia and P. Burattini, Progress in studying small-scale turbulence using exact two-point equations, New J. Phys 128 (2004), pp. 1-23

[30] P. Burattini, R.A. Antonia and L. Danaila, Similarity in the far field of a turbulent round jet, Phys. Fluids 17 (2005), 025101

[31] M.Z. Haq, C.G.W. Sheppard, R. Woolley, D.A. Greenhalgh and R.D. Lockett, Wrinkling and curvature of laminar and turbulent premixed flames, Comb. Flame 131 (2002), pp. 1-15

[32] B. Renou, A. Mura, E. Samson and A. Boukhalfa, Characterization of the local flame structure and the flame surface density in freely propagating premixed flames at various Lewis numbers, Combust. Sci. Tech. 174 (2002), pp. 143-179

[33] S. Candel and T. Poinsot, Flame stretch and the balance equation for the flame surface area, Comb. Sci. Tech. 70 (1990), pp. 1-15

[34] T. Poinsot, D. Veynante, Theoritical and Numerical Combustion (2001) Edwards.

[35] D. Bradley, P.H. Gaskell, X.J. Gu, Burning velocities, markstein lengths and flame quenching for spherical methane-air flames: a computational study Combust. Flame 104 (1996) 176-198.

[36] Y.Lafay, B. Renou, G. Cabot and M. Boukhalfa, Experimental and numerical investigation of the effect of H2 enrichment on laminar methane-air flame thickness, Comb. Flame 153 (2008), pp. 540-561

[37] M. Namazian, I.G. Shepherd and L. Talbot, Characterization of the density fluctuations in turbulent Vshaped premixed flames, Combust. Flame 64 (1986), 299

[38] A.N. Lipatnikov and J. Chomiak, Turbulent flame speed and thickness: phenomenology, evaluation and application in multi-dimensional simulations, Prog. Energy Comb. Sci. 28 (2002), pp. 1-74

[39] S. Corrsin, Estimates of the relation between Eulerian and Lagrangian scales in large Reynolds number turbulence, J. Atmosph. Sci. 20 (1963), pp. 115-119

[40] P. Goix, P. Paranthoën and M. Trinité, A tomographic study of measurements in a V-shaped $\mathrm{H}_{2}$-air flame and a Lagrangian interpretation of the turbulent flame brush evolution, Comb. Flame 81 (1988), pp. 229-241 